\title{
Columnar/Lamellar Packing in Cocrystals of Arylbipyridines with Diiodoperfluorobenzene
}

Remya Ramakrishnan, ${ }^{\perp}$ Ajith R. Mallia, ${ }^{\perp}$ Niyas M. A., Ramarani Sethy ${ }^{\dagger}$ and Mahesh Hariharan*

School of Chemistry, Indian Institute of Science Education and Research Thiruvananthapuram, CET

Campus, Sreekaryam, Thiruvananthapuram, Kerala, India 695016.

Supporting Information

\begin{tabular}{lll}
\hline Sl & Contents & Page \\
No: & & No: \\
\hline
\end{tabular}

1 Materials and methods.

2 Synthesis, procedure and characterization.

3 Table S1. Torsion angle between aryl and bipyridine units in the crystals and cocrystals. S7

$4 \quad$ Table S2. Represents packing efficiency and total packing energy of ArB crystals and S7 $\mathrm{ArB} \cdot \mathrm{D}$.

5 Table S3. Calculated topographical properties of the electron density function for the S7 intermolecular interactions in PhB, AnB, PhenB and PyB dimers.

6 Table S4. Interaction energy $\left(\mathrm{E}_{\mathrm{IQA}}\right)$ from Pendas' interacting quantum atoms approach S8 for $\mathrm{C}-\mathrm{H} \cdots \cdot \mathrm{H}-\mathrm{C}$ in $\mathrm{AnB}, \mathrm{F} \cdots \cdot \mathrm{F}$ and $\mathrm{I} \cdots \mathrm{I}$ in $(\mathrm{NaB})_{2} \cdot \mathrm{D}_{2.5}$.

7 Table S5. Percentage of intermolecular interactions present in ArB derivatives derived S9 from Hirshfeld surface analysis.

$8 \quad$ Table S6. Packing motifs in ArB derivatives.

9 Table S7. Calculated topographical properties of the electron density function for the S9 intermolecular interactions in $\mathrm{ArB} \cdot \mathrm{D}$ cocrystals.

10 Table S8. Percentage of intermolecular interactions present in $\mathrm{ArB} \cdot \mathrm{D}$ cocrystals derived $\mathrm{S} 12$ from Hirshfeld surface analyses.

11 Table S9. Packing motifs in ArB•D derivatives. $\quad$ S12

12 Figure S1. Torsion angle is measured across the atoms labelled in an asterisk (*). S13

13 Figure S2. QTAIM electron density maps of (a) PhB, (b) AnBa, (c) AnBb, (d) AnBc, S15 (e) PhenB, (f) PyBa and (g) PyBb. 
14 Figure S3. 2D-fingerprint plots representing $\mathrm{C}-\mathrm{H} \cdots \cdot \mathrm{N}$ interactions in crystalline (a) S16 $\mathrm{PhB}$, (b) AnB, (c) PhenB and (d) PyB obtained from Hirshfeld analyses.

15 Figure S4. 2D-fingerprint plots representing $\mathrm{C}-\mathrm{H} \cdot \bullet \mathrm{C}$ interactions in crystalline (a) S16 $\mathrm{PhB}$, (b) AnB, (c) PhenB and (d) PyB obtained from Hirshfeld analyses.

16 Figure S5. 2D-fingerprint plots representing $\mathrm{C}-\mathrm{H} \bullet \bullet \mathrm{H}-\mathrm{C}$ interactions in crystalline (a) S16 $\mathrm{PhB}$, (b) AnB, (c) PhenB and (d) PyB obtained from Hirshfeld analyses.

17 Figure S6. 2D-fingerprint plots representing $\mathrm{C} \cdot \bullet \mathrm{C}$ interactions in crystalline (a) $\mathrm{PhB}, \quad \mathrm{S} 16$ (b) AnB, (c) PhenB and (d) PyB obtained from Hirshfeld analyses.

18 Figure S7. Three dimensional pore distribution in $(\mathrm{NaB})_{2} \cdot \mathrm{D}_{2.5}$ cocrystal.

19 Figure S8. FT-IR spectra of $\mathrm{ArB}$ and $\mathrm{ArB} \cdot \mathrm{D}$. (a) $\mathrm{PhB}, \mathrm{PhB} \cdot \mathrm{D} 1$ and $\mathrm{PhB} \cdot \mathrm{D} 2$, (b) $\mathrm{NaB}, \mathrm{S} 17$ $(\mathrm{NaB})_{2} \cdot \mathrm{D}_{2.5}$ and $(\mathrm{NaB})_{3} \cdot \mathrm{D}_{2}$ and (c) PhenB and $\mathrm{PhenB} \cdot \mathrm{D}_{0.5}$.

20 Figure S9. FT-IR spectra of $\mathrm{ArB}$ and $\mathrm{ArB} \cdot \mathrm{D}$ and $\mathrm{D}$, (a) $\mathrm{PhB}, \mathrm{PhB} \cdot \mathrm{D} 1$ and $\mathrm{D}$, (b) $\mathrm{PhB}, \quad \mathrm{S} 18$ $\mathrm{PhB} \cdot \mathrm{D} 2$ and $\mathrm{D}$, (c) $\mathrm{NaB},(\mathrm{NaB})_{2} \cdot \mathrm{D}_{2.5}$ and $\mathrm{D}$, (d) $\mathrm{NaB},(\mathrm{NaB})_{3} \cdot \mathrm{D}_{2}$ and $\mathrm{D}$ and (e) PhenB and $\mathrm{PhenB} \cdot \mathrm{D}_{0.5}$ and $\mathrm{D}$.

21 Figure S10. QTAIM electron density maps of (a) PhB $\cdot \mathrm{D} 1 \mathrm{a}$, (b) $\mathrm{PhB} \cdot \mathrm{D} 1 \mathrm{~b}$, (c) $\mathrm{PhB} \cdot \mathrm{D} 2 \mathrm{a}, \quad \mathrm{S} 23$ (d) $\mathrm{PhB} \cdot \mathrm{D} 2 \mathrm{~b},(\mathrm{e})(\mathrm{NaB})_{2} \cdot \mathrm{D}_{2.5} \mathrm{a}$, (f) $(\mathrm{NaB})_{2} \cdot \mathrm{D}_{2.5} \mathrm{~b},(\mathrm{~g})(\mathrm{NaB})_{3} \cdot \mathrm{D}_{2}$, (h) PhenB $\bullet \mathrm{D}_{0.5}$ a and (i) PhenB $\cdot \mathrm{D}_{0.5} \mathrm{~b}$.

22 Figure S11. 2D-fingerprint plots representing $\mathrm{C}-\mathrm{H} \bullet \bullet \mathrm{F}$ interactions in crystalline (a) S24 $\mathrm{B} \cdot \mathrm{D}$, (b) $\mathrm{PhB} \cdot \mathrm{D} 1$, (c) $\mathrm{PhB} \cdot \mathrm{D} 2$, (d) $(\mathrm{NaB})_{2} \cdot \mathrm{D}_{2.5}$, (e) $(\mathrm{NaB})_{3} \cdot \mathrm{D}_{2}$ and (f) PhenB $\cdot \mathrm{D}_{0.5}$ obtained from Hirshfeld analyses.

23 Figure S12. 2D-fingerprint plots representing C-H $\bullet \bullet I$ interactions in crystalline (a) S24 $\mathrm{B} \cdot \mathrm{D}$, (b) $\mathrm{PhB} \cdot \mathrm{D} 1$, (c) $\mathrm{PhB} \cdot \mathrm{D} 2$, (d) $(\mathrm{NaB})_{2} \cdot \mathrm{D}_{2.5}$, (e) $(\mathrm{NaB})_{3} \cdot \mathrm{D}_{2}$ and (f) PhenB $\cdot \mathrm{D}_{0.5}$ obtained from Hirshfeld analyses.

24 Figure S13. 2D-fingerprint plots representing $\mathrm{C}-\mathrm{H} \cdot \cdots \mathrm{H}-\mathrm{C}$ interactions in crystalline (a) S25 $\mathrm{B} \cdot \mathrm{D}$, (b) $\mathrm{PhB} \cdot \mathrm{D} 1$, (c) $\mathrm{PhB} \cdot \mathrm{D} 2$, (d) $(\mathrm{NaB})_{2} \cdot \mathrm{D}_{2.5}$, (e) $(\mathrm{NaB})_{3} \cdot \mathrm{D}_{2}$ and (f) PhenB $\cdot \mathrm{D}_{0.5}$ obtained from Hirshfeld analyses.

25 Figure S14. Electrostatic surface potential (ESP) map of (a-d) ArB monomers, (f-i) S25 dimers and (j-n) cocrystals. ESP maps of (a) PhB, (b) AnB, (c) PhenB and (d) PyB monomers. ESP map of (e) D, (f) PhB, (g) AnB, (h) PhenB and (i) PyB dimers. ESP map of (j) $\mathrm{PhB} \cdot \mathrm{D} 1$, (k) $\mathrm{PhB} \cdot \mathrm{D} 2$, (l) $(\mathrm{NaB})_{2} \cdot \mathrm{D}_{2.5},(\mathrm{~m})(\mathrm{NaB})_{3} \cdot \mathrm{D}_{2}$ and (n) PhenB $\cdot \mathrm{D}_{0.5}$ cocrystals. 


\section{Materials and methods}

All chemicals were obtained from commercial suppliers and used as received without further purification. All reactions were carried out in oven-dried glassware prior to use and wherever necessary, were performed under dry nitrogen in dried, anhydrous solvents using standard gastight syringes, cannulas, and septa. Solvents were dried and distilled by standard procedures. TLC analysis were performed on precoated aluminium plates of silica gel 60 F254 plates $(0.25 \mathrm{~mm}$, Merck) and developed TLC plates were visualized under short and long wavelength UV lamps. Flash column chromatography was performed using silica gel of 200-400 mesh employing a solvent polarity correlated with the TLC mobility observed for the substance of interest. IR spectra for ArB derivatives and cocrystals were recorded on a Shimadzu IRPrestige-21 FT-IR spectrometer as KBr pellets. ${ }^{1} \mathrm{H}$ and ${ }^{13} \mathrm{C}$ NMR spectra were measured on a $500 \mathrm{MHz}$ Bruker advanced DPX spectrometer. Internal standard used for ${ }^{1} \mathrm{H}$ and ${ }^{13} \mathrm{C} \mathrm{NMR}$ is 1,1,1,1-tetramethyl silane (TMS). All the elemental analyses were performed on Elementar Vario MICRO Cube analyzer. All values recorded in elemental analyses are given in percentages. Reference standard used for elemental analysis is 4-aminobenzenesulphonic acid (sulphanilic acid).

X-ray crystallography: High-quality specimens of appropriate dimensions were selected for the X-ray diffraction experiments. Crystallographic data collected are presented in the supplementary information. Single crystals were mounted using oil (Infineum V8512) on a glass fibre. All measurements were made on a CCD area detector with graphite monochromated Mo $\mathrm{K} \alpha$ radiation $(\lambda=0.71073 \AA$ at $298 \mathrm{~K}$ ). The data was obtained using Bruker APEXII detector and processed using APEX2 from Bruker. All structures were solved by direct methods and expanded using Fourier techniques. The non-hydrogen atoms were refined anisotropically. Hydrogen atoms were included in idealized positions, but not refined. Their positions were constrained relative to their parent atom using the appropriate HFIX command in SHELXL97.The full validation of CIFs and structure factors of ArB derivatives and cocrystals were performed using Check CIF and found to be free from major alert level. 3D structure visualization and the exploration of the crystal packing of ArB derivatives and cocrystals were carried out using Mercury 3.8.

\section{Computational methods}

Quantum Theory of Atoms in Molecules (QTAIM) ${ }^{1}$ : The wave function for crystalline ArBs and were obtained employing the geometries taken from the crystal structure using Gaussian set of codes at B3LYP/6-311++G** level. ${ }^{2}$ For wave functions of cocrystalline ArB•Ds we employed a split basis method using GEN keyword. ${ }^{3}$ For carbon, hydrogen, nitrogen and fluorine B3LYP/6-311++G** level of theory was used while for iodine B3LYP/6-311G** level of theory was used. Different basis sets were collected from EMSL basis set exchange site. Quantum theory of atoms in molecules (QTAIM) analysis ${ }^{4}$ helps to understand the description of interatomic interaction in the single crystal X-ray structure. A bond is defined along the bond line between two nuclei, called a bond path, along which electron density is concentrated. The bond critical point $(\mathrm{BCP})$ is a point along the bond path at the interatomic surface, where the 
shared electron density reaches a minimum. The physical characteristics of the BCPs [the electron density at $\mathrm{BCP}, \rho\left(r_{\mathrm{BCP}}\right)$, and its Laplacian, $\left.\nabla^{2} \rho\left(r_{\mathrm{BCP}}\right)\right]$ reveal the approximate measure of the amount of electron density built up in the bonding region and as such could be taken as characteristic of the bond. When $\nabla^{2} \rho\left(r_{\mathrm{BCP}}\right)<0$ and is large in magnitude, $\rho\left(r_{\mathrm{BCP}}\right)$ is also large which means that there is a concentration of electronic charge in the internuclear region. This is also an indication of a sharing of electronic charge between both nuclei that defines the covalent (polar) bond. When $\nabla^{2} \rho\left(r_{\mathrm{BCP}}\right)>0$ there is a depletion of electronic charge in the internuclear region and it indicates a closed shell interaction. Using the AIM 2000 software package, the electron density was integrated over atomic basins according to the quantum theory of atoms in molecules using PROAIM, and thus the BCP data and the molecular graphs were obtained.

Interacting quantum atoms (IQA) ${ }^{4-6}$ approach was done using AIMALL software to understand the nature of intermolecular interactions. Hamiltonian is partitioned into physical atomic and interatomic contributions. The energy decomposition computed using partitioned Hamiltonian is consistent with the topological method of quantum theory of atoms in molecules. The interatomic energy contribution values determine the nature of intermolecular or intramolecular interactions.

Electrostatic Surface Potential ${ }^{7}$ : Electrostatic surface potential map illustrate the charge distributions of molecules three dimensionally. These maps allow us to visualize variably charged regions of a molecule. Knowledge of the charge distributions can be used to determine how molecules interact with one another. Gaussian supports the cube keyword to generate the cubes separately from the formatted checkpoint file using the cubegen utility program. This allows for the generation of electrostatic surface potential mapping of the molecule.

Hirshfeld Analysis ${ }^{8}$ : Important intermolecular interactions within the crystal structure of ArBs and $\mathrm{ArB} \bullet \mathrm{Ds}$ were identified through Hirshfeld surface analysis using Crystal Explorer 3.0. The Hirshfeld surface is defined as a set of points in 3D space where the ratio of promolecule and procrystal electron densities is equal to 0.5. The exploration of intermolecular contacts is provided by mapping normalized contact distances $\left(d_{\text {norm }}\right)$, which is a function of a closest distance from the point to the nuclei interior $\left(d_{\mathrm{i}}\right)$ and exterior $\left(d_{\mathrm{e}}\right)$ to the surface as well as on the van der Waals radii $\left(\mathrm{r}^{\mathrm{vdw}}\right)$. 2D fingerprint which were generated by deriving from the Hirshfeld surface by plotting the fraction of points on the surface as the function of $d_{\mathrm{i}}$ and $d_{\mathrm{e}}$ which provide a visual summary of intermolecular contacts within the crystal.

\section{Synthetic procedures}

General scheme for Suzuki coupling of 4-bromo-2, 2'-bipyridine' and aryl boronic acid: To a $0.86 \mathrm{mmol}$ of 4-bromo-2, 2'-bipyridine in dry THF, 2:1 aqueous $2 \mathrm{M} \mathrm{K}_{2} \mathrm{CO}_{3}$ was added under nitrogen atmosphere followed by respective equivalence of aryl boronic acid. The reaction mixture was then allowed to reflux at $70{ }^{0} \mathrm{C}$ after the addition of $\mathrm{Pd}\left(\mathrm{PPh}_{3}\right)_{4}$ as catalyst for 24 hours monitoring the progress of the reaction by means of TLC. The reaction mixture was then 
allowed to cool down to room temperature and extracted with Dichloromethane (DCM). The DCM layer was concentrated under vacuum and purified by means of (40\% ethyl acetatepetroleum ether) silica gel column chromatography. The ArB derivatives thus obtained were crystallized in DCM: Hexane (1:3) mixture.

PhB (yield=50\%). ${ }^{1} \mathrm{H}$ NMR $(500 \mathrm{MHz}$, DMSO-D 6 ): $\delta=8.83-8.80(\mathrm{~d}, \mathrm{~J}=6.50 \mathrm{~Hz}, 2 \mathrm{H}), 8.77-$ $8.76(\mathrm{~d}, \mathrm{~J}=3.5 \mathrm{~Hz}, 1 \mathrm{H}), 8.49-8.47(\mathrm{~d}, \mathrm{~J}=6.75 \mathrm{~Hz}, 2 \mathrm{H}), 8.12-8.10(\mathrm{~d}, \mathrm{~J}=8.00 \mathrm{~Hz}, 2 \mathrm{H}), 8.01-$ $8.00(\mathrm{~d}, \mathrm{~J}=7.50 \mathrm{~Hz}, 1 \mathrm{H}), 7.94(\mathrm{~s}, 1 \mathrm{H}), 7.61-7.60(\mathrm{t}, \mathrm{J}=7.00 \mathrm{~Hz}, 2 \mathrm{H}), 7.52-7.50$ (t, J =5.75 Hz, $1 \mathrm{H}) ;{ }^{13} \mathrm{C}$ NMR $(125 \mathrm{MHz}$, DMSO-D 6 ): $\delta=156.42,155.56,150.53,149.80,148.76,137.90$, 137.77, 129.89, 129.81, 127.33, 124.85, 122.11, 121.16, 118.13; IR (KBr): 3053, 2999, 2997, 1577, 1543, 1454, 1386, 796, 759, 682, $613 \mathrm{~cm}^{-1}$; HR-MS (EI)-(m/z): 232.1001. Calcd. for $\mathrm{C}_{16} \mathrm{H}_{12} \mathrm{~N}_{2}$ : 232.1000; Elemental analysis: (\%) Calcd. for $\mathrm{C}_{16} \mathrm{H}_{12} \mathrm{~N}_{2}: \mathrm{C}, 82.73 ; \mathrm{H}, 5.21 ; \mathrm{N}, 12.06$. Found: C, 82.72; H, 5.15; N, 12.07 .

NaB (yield = 43.6\%). ${ }^{1} \mathrm{H}$ NMR $\left(500 \mathrm{MHz}, \mathrm{DMSO}_{-} \mathrm{D}_{6}\right): \delta=8.83-8.80(\mathrm{~d}, \mathrm{~J}=6.50 \mathrm{~Hz}, 2 \mathrm{H}), 8.77$ - $8.76(\mathrm{~d}, \mathrm{~J}=3.5 \mathrm{~Hz}, 1 \mathrm{H}), 8.49-8.47(\mathrm{~d}, \mathrm{~J}=6.75 \mathrm{~Hz}, 2 \mathrm{H}), 8.12-8.10(\mathrm{~d}, \mathrm{~J}=8.00 \mathrm{~Hz}, 2 \mathrm{H}), 8.01$ $8.00(\mathrm{~d}, \mathrm{~J}=7.50 \mathrm{~Hz}, 3 \mathrm{H}), 7.94(\mathrm{~s}, 1 \mathrm{H}), 7.61-7.60(\mathrm{t}, \mathrm{J}=7.00 \mathrm{~Hz}, 2 \mathrm{H}), 7.52-7.50(\mathrm{t}, \mathrm{J}=5.75 \mathrm{~Hz}$, $1 \mathrm{H}) ;{ }^{13} \mathrm{C}$ NMR $(125 \mathrm{MHz}$, DMSO-D 6 ): $\delta=137.89,135.07,133.65,133.64,129.44,129.10$, 128.06, 127.51, 127.22, 126.74, 124.88, 122.33, 121.19, 118.31; IR (KBr): 3437, 3051, 3003, 1581, 1454, 1394, 823, $793 \mathrm{~cm}^{-1}$; HR-MS (EI)-(m/z): 282.1160. Calcd. for $\mathrm{C}_{20} \mathrm{H}_{14} \mathrm{~N}_{2}: 282.1157$; Elemental analysis: (\%) Calcd. for $\mathrm{C}_{20} \mathrm{H}_{14} \mathrm{~N}_{2}$ : C, 85.08; H, 5.00; N, 9.92. Found: C, 85.2; H, $4.85 ; \mathrm{N}, 9.97$. NaB doesn't yield single crystals for characterisation using X-ray diffraction.

AnB (yield = 45.8\%). ${ }^{1} \mathrm{H}$ NMR $\left(500 \mathrm{MHz}, \mathrm{DMSO}^{-} \mathrm{D}_{6}\right): \delta=8.97-8.96(\mathrm{~d}, \mathrm{~J}=4.65 \mathrm{~Hz}, 1 \mathrm{H}), 8.79$ $(\mathrm{s}, 1 \mathrm{H}), 8.62-8.61(\mathrm{~d}, \mathrm{~J}=3.90 \mathrm{~Hz}, 1 \mathrm{H}), 8.58-8.56(\mathrm{~d}, \mathrm{~J}=7.8 \mathrm{~Hz}, 1 \mathrm{H}), 8.41(\mathrm{~s}, 1 \mathrm{H}), 8.22-8.20$ $(\mathrm{d}, \mathrm{J}=8.60 \mathrm{~Hz}, 2 \mathrm{H}), 8.04-8.01(\mathrm{t}, \mathrm{J}=7.72 \mathrm{~Hz}, 1 \mathrm{H}), 7.58-7.56(\mathrm{t}, \mathrm{J}=6.90 \mathrm{~Hz}, 5 \mathrm{H}), 7.49-7.47$ (t, J $=7.00 \mathrm{~Hz}, 3 \mathrm{H}) ;{ }^{13} \mathrm{C}$ NMR (125 MHz, DMSO-D $\left.{ }_{6}\right): \delta=137.96,133.81,131.27,129.22$, 129.12, 128.05, 127.03, 126.99, 125.99, 125.82, 124.95, 123.09, 121.25; IR (KBr): 3049, 1581, 1537, 1458, 1387, 881, 791, 739, $652 \mathrm{~cm}^{-1}$; HR-MS (EI)-(m/z): 332.1320. Calcd. for $\mathrm{C}_{24} \mathrm{H}_{16} \mathrm{~N}_{2}$ : 332.1313; Elemental analysis: (\%) Calcd. for $\mathrm{C}_{24} \mathrm{H}_{16} \mathrm{~N}_{2}$ : C, 86.72; H, 4.85; N, 8.43. Found: C, 86.89; H, 4.83; N, 8.49.

PhenB (yield = 90\%). ${ }^{1} \mathrm{H}$ NMR $\left(500 \mathrm{MHz}, \mathrm{DMSO}_{-} \mathrm{D}_{6}\right): \delta=8.83-8.80(\mathrm{~d}, \mathrm{~J}=6.50 \mathrm{~Hz}, 2 \mathrm{H})$, $8.77-8.76(\mathrm{~d}, \mathrm{~J}=3.5 \mathrm{~Hz}, 2 \mathrm{H}), 8.49-8.47(\mathrm{~d}, \mathrm{~J}=6.75 \mathrm{~Hz}, 2 \mathrm{H}), 8.12-8.10(\mathrm{~d}, \mathrm{~J}=8.00 \mathrm{~Hz}, 2 \mathrm{H})$, $8.01-8.00(\mathrm{~d}, \mathrm{~J}=7.50 \mathrm{~Hz}, 3 \mathrm{H}), 7.94(\mathrm{~s}, 2 \mathrm{H}), 7.61-7.60(\mathrm{t}, \mathrm{J}=7.00 \mathrm{~Hz}, 2 \mathrm{H}), 7.52-7.50(\mathrm{t}, \mathrm{J}$ $=5.75 \mathrm{~Hz}, 1 \mathrm{H}) ;{ }^{13} \mathrm{C}$ NMR $(125 \mathrm{MHz}$, DMSO-D 6 ): $\delta=156.00,155.47,149.84,149.34,137.96$, 136.02, 131.27, 130.69, 130.24, 129.67, 129.48, 128.22, 128.11, 127.81, 127.68, 126.09, 125.65, 124.92, 124.10, 123.34, 121.76, 121.2; IR (KBr): 3049, 3007, 2920, 2850, 1579, 1539, 1454 , 1382, 792, 744, $655 \mathrm{~cm}^{-1}$; (m/z): HR-MS (EI)-(m/z): 332.1324. Calcd. for $\mathrm{C}_{24} \mathrm{H}_{16} \mathrm{~N}_{2}: 332.1313$; Elemental analysis: (\%) Calcd. for $\mathrm{C}_{24} \mathrm{H}_{16} \mathrm{~N}_{2}: \mathrm{C}, 86.72 ; \mathrm{H}, 4.85 ; \mathrm{N}, 8.43$. Found: $\mathrm{C}, 86.76 ; \mathrm{H}$, $4.83 ; \mathrm{N}, 8.49$. 
PyB (yield = 37.46\%). ${ }^{1} \mathrm{H}$ NMR (500 MHz, DMSO-D $): \delta=8.91-8.90(\mathrm{~d}, \mathrm{~J}=5.00 \mathrm{~Hz}, 1 \mathrm{H})$, $8.69-8.68(\mathrm{~d}, \mathrm{~J}=4.50 \mathrm{~Hz}, 1 \mathrm{H}), 8.65(\mathrm{~s}, 1 \mathrm{H}), 8.55-8.53(\mathrm{~d}, \mathrm{~J}=8.00 \mathrm{~Hz}, 1 \mathrm{H}), 8.44-8.42(\mathrm{~d}, \mathrm{~J}=$ $8.00 \mathrm{~Hz}, 1 \mathrm{H}), 8.38-8.37$ (d, J = 7.50Hz, 1H), 8.33 - 8.32 (d, J = 7.50 Hz, 1H), 8.27 (s,2H), 8.24 - $8.22(\mathrm{~d}, \mathrm{~J}=9.00 \mathrm{~Hz}, 1 \mathrm{H}), 8.15-8.11(\mathrm{~m}, 2 \mathrm{H}), 8.03$ - $8.00(\mathrm{~m}, 1 \mathrm{H}), 7.76-7.75$ (d, J = 3.50 Hz, 1H), 7.50 - $7.48(\mathrm{~m}, 2 \mathrm{H}) ;{ }^{13} \mathrm{C}$ NMR (125 MHz, DMSO-D $): \delta=137.94,134.70,131.46,131.38$, 130.78, 128.96, 128.54, 127.88, 127.82, 127.77, 127.11, 126.28, 126.09, 125.89, 125.63, 124.91, 124.55, 124.37, 124.30, 122.24, 121.24; IR (KBr): 3439, 3039, 1583, 1460, 1390, 844, 793, 719 $\mathrm{cm}^{-1}$; HR-MS (EI)-(m/z): 356.3212. Calcd. for $\mathrm{C}_{26} \mathrm{H}_{16} \mathrm{~N}_{2}$ : 356.3245; Elemental analysis: (\%) Calcd. for $\mathrm{C}_{26} \mathrm{H}_{16} \mathrm{~N}_{2}$ : C, 87.62; H, 4.52; N, 7.86. Found: C, 87.69; H, 4.59; N, 7.90.

General procedure for the synthesis of the cocrystals: 1,4-diiodotetrafluorobenzene (D) with $\mathrm{PhB}$ and PhenB derivatives were dissolved in 1:1 stoichiometric ratio in 1:3 chloroform:hexane solvent mixture to obtain cocystalline $\mathrm{PhB} \cdot \mathrm{D} 1$ and $\mathrm{PhenB} \cdot \mathrm{D}_{0.5}$. Cocrystals of $\mathrm{PhB} \cdot \mathrm{D} 2$ polymorph was obtained when $\mathrm{D}$ and $\mathrm{PhB}$ were dissolved in 1:1 stoichiometric ratio in 1:3 dichloromethane:hexane solvent mixture. $(\mathrm{NaB})_{2} \bullet \mathrm{D}_{2.5}$ and $(\mathrm{NaB})_{3} \bullet \mathrm{D}_{2}$ got crystallized when $\mathrm{NaB}$ and $\mathrm{D}$ were mixed in $1: 1$ and $3: 2$ stoichiometric ratio respectively in 1:3 chloroform:hexane solvent mixture. All the crystals were isolated prior to the complete evaporation of the solvent mixtures to avoid precipitation of impurities. Yield of the cocrystals was calculated based on ArB derivatives. PhB•D1 (Yield = 87\%): IR $(\mathrm{KBr}): 3057,3005,1583,1462,941,758,694 \mathrm{~cm}^{-1}$. Elemental analysis: (\%) Calcd. for $\mathrm{C}_{22} \mathrm{H}_{12} \mathrm{~F}_{4} \mathrm{I}_{2} \mathrm{~N}_{2}$ : C, 41.67; H, 1.91; N, 4.42. Found: C, 41.31; $\mathrm{H}$, 1.83; N, 4.40. PhB•D2 (Yield = 83\%): IR $(\mathrm{KBr}): 3055,3011,1585,1464,943,760,696 \mathrm{~cm}^{-1}$. Elemental analysis: (\%) Calcd. for $\mathrm{C}_{22} \mathrm{H}_{12} \mathrm{~F}_{4} \mathrm{I}_{2} \mathrm{~N}_{2}$ : C, 41.67; H, 1.91; N, 4.42. Found: C, 41.50; $\mathrm{H}$, 1.62; N, 4.74. $(\mathrm{NaB})_{2} \bullet \mathrm{D}_{2.5}$ (Yield = 62\%): IR $(\mathrm{KBr}): 3053,3013,1583,1466,941,756 \mathrm{~cm}^{-1}$. Elemental analysis: (\%) Calcd. for $\mathrm{C}_{55} \mathrm{H}_{28} \mathrm{~F}_{10} \mathrm{I}_{5} \mathrm{~N}_{4}$ : C, 42.09; H, 1.80; N, 3.57. Found: C, 42.31; $\mathrm{H}, 1.83 ; \mathrm{N}, 3.40 .(\mathrm{NaB})_{3} \bullet \mathrm{D}_{2}($ Yield $=89 \%): \mathrm{IR}(\mathrm{KBr}): 3053,3015,1583,1462,943,790,741$ $\mathrm{cm}^{-1}$. Elemental analysis: (\%) Calcd. for $\mathrm{C}_{72} \mathrm{H}_{42} \mathrm{~F}_{8} \mathrm{I}_{4} \mathrm{~N}_{6}$ : C, 52.39; H, 2.56; N, 5.09. Found: C, 51.91; H, 1.97; N, 4.61. PhenB• $\mathrm{D}_{0.5}($ Yield = 96\%): IR (KBr): 3061, 3012, 1587, 1462, 943, 760, $717 \mathrm{~cm}^{-1}$. Elemental analysis: (\%) Calcd. for $\mathrm{C}_{27} \mathrm{H}_{16} \mathrm{~F}_{2} \mathrm{IN}_{2}: \mathrm{C}, 60.80 ; \mathrm{H}, 3.02 ; \mathrm{N}, 5.25$ Found: C, $60.69 ; \mathrm{H}, 2.93 ; \mathrm{N}, 5.14$. 


\begin{tabular}{|c|c|c|c|c|c|}
\hline $\mathrm{PhB}$ & N30-H34 & 2.654919 & 0.007611 & 0.022906 & 5.106597 \\
\hline \multirow[t]{10}{*}{$\mathrm{AnBa}$} & N42-H45 & 2.731114 & 0.006696 & 0.01945 & 4.39771 \\
\hline & H31-C46 & 2.859489 & 0.005112 & 0.015515 & 3.09809 \\
\hline & C39-N83 & 3.435588 & 0.004798 & 0.013737 & 2.95369 \\
\hline & N42-C69 & 3.472243 & 0.004639 & 0.013169 & 2.82241 \\
\hline & C16-C52 & 3.714692 & 0.003636 & 0.009966 & 2.07415 \\
\hline & C18-C43 & 3.707167 & 0.003689 & 0.009582 & 2.03476 \\
\hline & $\mathrm{C} 22-\mathrm{C} 46$ & 3.806261 & 0.003136 & 0.008687 & 1.79847 \\
\hline & C14-C50 & 3.742965 & 0.003356 & 0.008601 & 1.90349 \\
\hline & C13-C48 & 3.773439 & 0.003332 & 0.008538 & 1.83785 \\
\hline & H21-H68 & 2.731068 & 0.002275 & 0.007961 & 1.52279 \\
\hline \multirow[t]{3}{*}{$\mathrm{AnBb}$} & N41-H78 & 3.133821 & 0.002865 & 0.009258 & 1.96913 \\
\hline & H34-H76 & 2.245033 & 0.004835 & 0.014885 & 3.22937 \\
\hline & H36-N83 & 3.133881 & 0.002865 & 0.009257 & 1.96913 \\
\hline $\mathrm{AnBc}$ & C35 - C77 & 3.391626 & 0.004889 & 0.016429 & 2.83554 \\
\hline \multirow[t]{5}{*}{ PhenB } & H40-N83 & 2.70173 & 0.006442 & 0.018484 & 4.18767 \\
\hline & $\mathrm{C} 16-\mathrm{C} 45$ & 3.499383 & 0.004894 & 0.013453 & 2.70427 \\
\hline & H37-H54 & 2.815951 & 0.001690 & 0.00608 & 7.98152 \\
\hline & C36-C62 & 3.509563 & 0.005285 & 0.013422 & 2.87492 \\
\hline & C32-C66 & 3.541205 & 0.004829 & 0.012527 & 2.66488 \\
\hline PyBa & C39-H84 & 2.815982 & 0.005297 & 0.015111 & 3.16373 \\
\hline \multirow[t]{5}{*}{ PyBb } & H16 - C49 & 2.987195 & 0.003941 & 0.011672 & 2.52048 \\
\hline & H16 - C68 & 3.085031 & 0.003931 & 0.011453 & 2.50735 \\
\hline & H14 - C49 & 3.33116 & 0.002373 & 0.006082 & 1.31275 \\
\hline & C29 - H46 & 4.294172 & 0.000391 & 0.001315 & 0.15753 \\
\hline & H30 - N88 & 2.65338 & 0.007161 & 0.021029 & 4.71277 \\
\hline
\end{tabular}

${ }^{a} d=$ distance, ${ }^{b} \rho(r)=$ electron density at $B C P,{ }^{c} \nabla^{2} \rho(r)=$ Laplacian of electron density at BCP, ${ }^{d} d E=$ dissociation energy. $a, b$ and $c$ indicate different dimers of same molecule.

Table S4. Interaction energy ( $\left.\mathrm{E}_{\mathrm{IQA}}\right)$ from Pendas' interacting quantum atoms approach for C$\mathrm{H} \bullet \bullet \mathrm{H}-\mathrm{C}$ in $\mathrm{AnB}$, F $\bullet \bullet F$ and $\mathrm{I} \bullet \bullet \mathrm{I}$ in $(\mathrm{NaB})_{2} \bullet \mathrm{D}_{2.5} \cdot \mathrm{E}_{\mathrm{CIQA}}$ corresponds to Coulombic part of the interaction energy. Values are in $\mathrm{kcal} / \mathrm{mol}$.

\begin{tabular}{ccc}
\hline Interaction & $\mathrm{E}_{\mathrm{IQA}}$ & $\mathrm{E}_{\mathrm{CIQA}}$ \\
\hline $\mathrm{C}-\mathrm{H} \bullet \bullet \cdot \mathrm{H}-\mathrm{C}$ & -0.17 & 1.51 \\
$\mathrm{~F} \bullet \bullet \cdot \mathrm{F}$ & 33.01 & 40.16 \\
$\mathrm{I} \bullet \bullet \mathrm{I}$ & 2.67 & 3.91 \\
\hline
\end{tabular}


Table S5. Percentage of intermolecular interactions present in ArB derivatives derived from Hirshfeld surface analysis.

\begin{tabular}{ccccc}
\hline Interactions & PhB $(\%)$ & AnB (\%) & PhenB (\%) & PyB (\%) \\
\hline C-H & 30.1 & 32.4 & 37.1 & 20.6 \\
H-H & 48.2 & 51.1 & 47.4 & 54.3 \\
N-H & 13.0 & 8.3 & 9.4 & 6.9 \\
C-C & 8.6 & 6.0 & 5.7 & 15.3 \\
C-N & 0.1 & 2.2 & 0.3 & 2.9 \\
\hline
\end{tabular}

Table S6. Packing motifs in ArB derivatives.

\begin{tabular}{ccccc}
\hline Entry & \% C-H & \% C-C & $\rho=\% \mathbf{C}-\mathbf{H} / \% \mathbf{C}-\mathbf{C}$ & Motif \\
\hline PhB & 30.1 & 8.6 & 3.5 & Sandwich-herringbone \\
AnB & 32.4 & 6.0 & 5.4 & Herringbone \\
PhenB & 37.1 & 5.7 & 6.5 & Herringbone \\
PyB & 20.6 & 15.3 & 1.3 & $\gamma$ \\
\hline
\end{tabular}

Table S7. Calculated topographical properties of the electron density function for the intermolecular interactions in cocrystals.

\begin{tabular}{cccccc}
\hline Entry & Interactions & ${ }^{\mathbf{a}} \mathbf{d}(\mathbf{A})$ & ${ }^{\mathbf{b}} \boldsymbol{\rho}(\mathbf{r})(\mathbf{a} \cdot \mathbf{u})$. & ${ }^{\mathbf{c}} \nabla^{2} \boldsymbol{\rho}(\mathbf{r})(\mathbf{a} . \mathbf{u .})$ & ${ }^{\mathbf{d}} \Delta \mathbf{E}(\mathbf{k J} / \mathbf{m o l})$ \\
\hline PhB•D1a & N29 - I41 & 3.095293 & 0.015985 & 0.042118 & 11.43274 \\
& F37 - H46 & 2.502882 & 0.006928 & 0.031233 & 6.712091 \\
& H22 - I41 & 3.119038 & 0.006212 & 0.018803 & 3.824041 \\
& H24 - H48 & 2.590898 & 0.003668 & 0.012949 & 2.4916 \\
\hline PhB•D1b & I12 - N72 & 3.162608 & 0.013892 & 0.037010 & 9.707786 \\
& F8 - H18 & 2.557228 & 0.005547 & 0.022462 & 4.975323 \\
& I12 - H56 & 3.136754 & 0.005743 & 0.016513 & 3.421027 \\
& F9 - H50 & 2.849468 & 0.003494 & 0.015907 & 3.014074 \\
& C23 - H68 & 3.108339 & 0.003958 & 0.012183 & 2.399707 \\
& H31 - H68 & 2.909731 & 0.002981 & 0.010913 & 1.924492 \\
& I12 - H18 & 3.827583 & 0.002577 & 0.007428 & 1.206417 \\
& C17 - H70 & 3.429542 & 0.002387 & 0.007392 & 1.436149 \\
& I12 - H52 & 3.804989 & 0.002312 & 0.006858 & 1.136842 \\
\hline PhB•D2a & I41 - N72 & 2.937019 & 0.021634 & 0.056462 & 16.69293
\end{tabular}




\begin{tabular}{|c|c|c|c|c|c|}
\hline \multicolumn{2}{|c|}{$\mathrm{H} 24-\mathrm{F} 40$} & 2.576862 & 0.005910 & 0.025593 & 5.554245 \\
\hline & $\mathrm{I} 42-\mathrm{C} 73$ & 3.438969 & 0.008769 & 0.023195 & 5.06459 \\
\hline & F38 - H110 & 2.581365 & 0.005208 & 0.021852 & 4.77841 \\
\hline & $\mathrm{H} 26-\mathrm{F} 40$ & 2.739020 & 0.004165 & 0.020000 & 3.994698 \\
\hline & I41 - H60 & 3.248240 & 0.005291 & 0.017448 & 3.335698 \\
\hline & H46 - H106 & 2.710856 & 0.003183 & 0.012090 & 2.204107 \\
\hline & F37 - H108 & 2.884041 & 0.002717 & 0.011902 & 2.285498 \\
\hline & H48 - H106 & 2.705795 & 0.002871 & 0.010295 & 1.936306 \\
\hline & H46 - H108 & 2.705795 & 0.002849 & 0.010267 & 1.924492 \\
\hline & I42 - H86 & 3.511493 & 0.003351 & 0.009778 & 1.906113 \\
\hline & H26 - H70 & 2.776977 & 0.002597 & 0.009090 & 1.639625 \\
\hline & H28 - H68 & 2.776977 & 0.002598 & 0.009041 & 1.625185 \\
\hline & I41 - H44 & 3.540382 & 0.003255 & 0.008640 & 1.751209 \\
\hline & F37 - H44 & 3.139418 & 0.001620 & 0.007768 & 1.198541 \\
\hline & H26 - I41 & 3.785873 & 0.002103 & 0.006064 & 1.063328 \\
\hline \multirow[t]{7}{*}{$\mathrm{PhB} \cdot \mathrm{D} 2 \mathrm{~b}$} & I12 - N71 & 3.04966 & 0.017454 & 0.046425 & 12.832131 \\
\hline & I11 - N42 & 3.08212 & 0.016116 & 0.043378 & 11.749113 \\
\hline & F10 - H20 & 2.53385 & 0.006573 & 0.02993 & 6.431162 \\
\hline & $\mathrm{F} 10-\mathrm{H} 22$ & 2.70506 & 0.004876 & 0.023594 & 4.725900 \\
\hline & I12 - H64 & 3.05683 & 0.006741 & 0.020176 & 4.148290 \\
\hline & I11 - H30 & 3.18919 & 0.005344 & 0.015727 & 3.210987 \\
\hline & I11 - H22 & 3.73040 & 0.002431 & 0.007129 & 1.258927 \\
\hline \multirow[t]{8}{*}{$(\mathrm{NaB})_{2} \cdot \mathrm{D}_{2.5} \mathrm{a}$} & N35 - I48 & 3.098936 & 0.015797 & 0.042219 & 11.38679 \\
\hline & I47 - N84 & 3.123438 & 0.015152 & 0.040360 & 10.79081 \\
\hline & F43 - H52 & 2.669393 & 0.004364 & 0.019173 & 4.035394 \\
\hline & H4 - F45 & 2.726210 & 0.003853 & 0.017258 & 3.553614 \\
\hline & I47 - H82 & 3.191714 & 0.005239 & 0.015804 & 3.195234 \\
\hline & H34 - I48 & 3.211695 & 0.005090 & 0.015700 & 3.134847 \\
\hline & I47 - H50 & 3.356391 & 0.004607 & 0.012015 & 2.532295 \\
\hline & H2 - I48 & 3.412112 & 0.004156 & 0.010911 & 2.269745 \\
\hline \multirow[t]{6}{*}{$(\mathrm{NaB})_{2} \cdot \mathrm{D}_{2.5} \mathrm{~b}$} & F10 - F17 & 2.514614 & 0.013094 & 0.067996 & 17.00143 \\
\hline & I12 - N59 & 3.098936 & 0.015809 & 0.042092 & 11.36448 \\
\hline & F16 - H31 & 2.609216 & 0.004767 & 0.018932 & 4.166669 \\
\hline & F9 - H28 & 2.726292 & 0.003838 & 0.017219 & 3.5418 \\
\hline & I12 - H58 & 3.211639 & 0.005080 & 0.015700 & 3.132222 \\
\hline & I12 - H26 & 3.412020 & 0.004150 & 0.010920 & 2.271058 \\
\hline
\end{tabular}




\begin{tabular}{|c|c|c|c|c|c|}
\hline & I18 - H33 & 3.652072 & 0.002698 & 0.008076 & 1.447963 \\
\hline & I18 - H31 & 3.783547 & 0.002233 & 0.007179 & 1.195915 \\
\hline & I11 - I24 & 5.254908 & 0.001089 & 0.002542 & 0.347879 \\
\hline \multirow{12}{*}{$(\mathrm{NaB})_{3} \cdot \mathrm{D}_{2} \mathrm{a}$} & $\mathrm{H} 12-\mathrm{H} 20$ & 2.034910 & 0.011956 & 0.056185 & 10.66478 \\
\hline & N71 - I83 & 3.139970 & 0.014589 & 0.039072 & 10.3576 \\
\hline & H17 - F81 & 2.581125 & 0.006335 & 0.028287 & 6.1056 \\
\hline & H15 - F81 & 2.632348 & 0.006236 & 0.027586 & 5.907375 \\
\hline & H68 - F79 & 2.777853 & 0.004797 & 0.021233 & 4.208677 \\
\hline & H48 - I83 & 3.078914 & 0.006226 & 0.018055 & 3.763654 \\
\hline & H34 - F82 & 2.803067 & 0.003138 & 0.013199 & 2.720018 \\
\hline & $\mathrm{H} 29-\mathrm{H} 40$ & 2.581014 & 0.003409 & 0.011119 & 2.242177 \\
\hline & H15 - F82 & 3.029971 & 0.002157 & 0.010022 & 1.673756 \\
\hline & H70 - I83 & 3.567329 & 0.003493 & 0.010009 & 1.854916 \\
\hline & H31 - H38 & 3.087552 & 0.001042 & 0.004429 & 0.681317 \\
\hline & H31 - I83 & 4.411298 & 0.000667 & 0.002116 & 0.26255 \\
\hline \multirow[t]{8}{*}{ PhenB $\bullet D_{0.5} \mathrm{a}$} & I54 - N96 & 3.080519 & 0.016445 & 0.043964 & 11.94734 \\
\hline & N42 - I48 & 3.080519 & 0.016445 & 0.043963 & 11.94734 \\
\hline & I54 - H66 & 3.142797 & 0.005640 & 0.017358 & 3.48929 \\
\hline & H12 - I48 & 3.142811 & 0.005640 & 0.017358 & 3.48929 \\
\hline & F52 - C90 & 3.524132 & 0.002634 & 0.010864 & 1.79978 \\
\hline & C36 - F46 & 3.524195 & 0.002634 & 0.010863 & 1.79978 \\
\hline & I54 - H94 & 3.601461 & 0.003209 & 0.008965 & 1.671131 \\
\hline & $\mathrm{H} 40$ - I48 & 3.601418 & 0.003209 & 0.008965 & 1.672444 \\
\hline \multirow[t]{8}{*}{ PhenB $\bullet D_{0.5} b$} & $\mathrm{~F} 4-\mathrm{H} 49$ & 2.652997 & 0.004662 & 0.020561 & 4.366207 \\
\hline & F10 - H91 & 2.652997 & 0.004662 & 0.020560 & 4.366207 \\
\hline & F10 - H89 & 2.901296 & 0.002733 & 0.014269 & 2.588743 \\
\hline & F4 - H47 & 2.901372 & 0.002732 & 0.014268 & 2.588743 \\
\hline & I12 - H91 & 3.790635 & 0.002047 & 0.005999 & 1.030509 \\
\hline & I6 - H49 & 3.790676 & 0.002047 & 0.005999 & 1.030509 \\
\hline & I12 - H94 & 4.118189 & 0.001056 & 0.003318 & 0.45815 \\
\hline & I6 - H52 & 4.118251 & 0.001056 & 0.003317 & 0.456837 \\
\hline
\end{tabular}

${ }^{\mathrm{a}} \mathrm{d}=$ distance, ${ }^{\mathrm{b}} \rho(\mathrm{r})=$ electron density at $\mathrm{BCP},{ }^{\mathrm{c}} \nabla^{2} \rho(\mathrm{r})=$ Laplacian of electron density at BCP, ${ }^{\mathrm{d}} \Delta \mathrm{E}=$ dissociation energy. 
Table S8. Percentage of intermolecular interactions present in $\mathrm{ArB} \cdot \mathrm{D}$ cocrystals derived from Hirshfeld surface analysis.

\begin{tabular}{|c|c|c|c|c|c|c|}
\hline Interactions & $\mathbf{B} \cdot \mathbf{D}$ & PhB•D1(\%) & $\mathrm{PhB} \cdot \mathrm{D} 2(\%)$ & $(\mathrm{NaB})_{2} \cdot \mathrm{D}_{2.5}(\%)^{\mathrm{a}}$ & $(\mathrm{NaB})_{3} \cdot \mathrm{D}_{2}(\%)$ & PhenB $\cdot D_{0.5}(\%)$ \\
\hline $\mathrm{C}-\mathrm{H}$ & 12.9 & 22 & 11.4 & 7.1 & 10.9 & 9.5 \\
\hline $\mathrm{C}-\mathrm{C}$ & 3.9 & 5.4 & 9.6 & 6.9 & 11.7 & 15 \\
\hline $\mathrm{H}-\mathrm{H}$ & 15.7 & 14.2 & 18.8 & 19.1 & 38.9 & 34.4 \\
\hline $\mathrm{H}-\mathrm{F}$ & 11.8 & 22 & 19.7 & 19.8 & 14.9 & 18.5 \\
\hline $\mathrm{N}-\mathrm{H}$ & 5.6 & 3.3 & 3.2 & 0.5 & 3.4 & 2.9 \\
\hline $\mathrm{C}-\mathrm{N}$ & 0.5 & 1.3 & 1.8 & 2.6 & 3.4 & 2.7 \\
\hline H-I & 17 & 15.3 & 14.6 & 16.6 & 9.9 & 7.2 \\
\hline $\mathrm{N}-\mathrm{N}$ & 0 & 0 & 0 & 0.1 & 0 & 0.1 \\
\hline $\mathrm{C}-\mathrm{F}$ & 10.3 & 7 & 5.9 & 4.8 & 2.7 & 3.4 \\
\hline C-I & 4.3 & 0.8 & 4.7 & 2.5 & 1.9 & 2.4 \\
\hline N-I & 4.1 & 1.6 & 3.4 & 4.9 & 1.4 & 1.5 \\
\hline F-F & 8.6 & 3.4 & 3 & 4.9 & 0.4 & 0.3 \\
\hline F-I & 5 & 1.8 & 3.1 & 0.9 & & 1.1 \\
\hline $\mathrm{N}-\mathrm{F}$ & 0.3 & 1.1 & 0.8 & 0 & 0.5 & 0 \\
\hline I-I & 0 & 0.7 & 0.2 & 0.9 & 0 & 0 \\
\hline
\end{tabular}

${ }^{a}$ sum of all the interactions in $(\mathrm{NaB})_{2} \bullet \mathrm{D}_{2.5}{ }^{\mathrm{a}}$ is estimated to be $91.6 \%$. Void space present in the $(\mathrm{NaB})_{2} \bullet \mathrm{D}_{2.5}$ accounts for the remaining $8.4 \%$, further confirming the porous nature.

Table S9. Packing motifs in $\mathrm{ArB} \cdot \mathrm{D}$ cocrystals.

\begin{tabular}{ccccc}
\hline & \% C-H & \% C-C & $\boldsymbol{\rho}=\boldsymbol{\%} \mathbf{C}-\mathbf{H} / \% \mathbf{C}-\mathbf{C}$ & Motif \\
\hline $\mathrm{B} \bullet \mathrm{D}$ & 12.9 & 3.9 & 3.31 & Sandwich-herringbone \\
$\mathrm{PhB} \cdot \mathrm{D} 1$ & 22 & 5.4 & 4.07 & Sandwich-herringbone \\
$\mathrm{PhB} \cdot \mathrm{D} 2$ & 11.4 & 9.6 & 1.19 & $\gamma$ \\
$(\mathrm{NaB})_{2} \cdot \mathrm{D}_{2.5}$ & 7.1 & 6.9 & 1.03 & $\beta$ \\
$(\mathrm{NaB})_{3} \cdot \mathrm{D}_{2}$ & 10.9 & 11.7 & 0.93 & $\beta$ \\
$\mathrm{PhenB} \cdot \mathrm{D}_{0.5}$ & 9.5 & 15 & 0.63 & $\beta$ \\
\hline
\end{tabular}



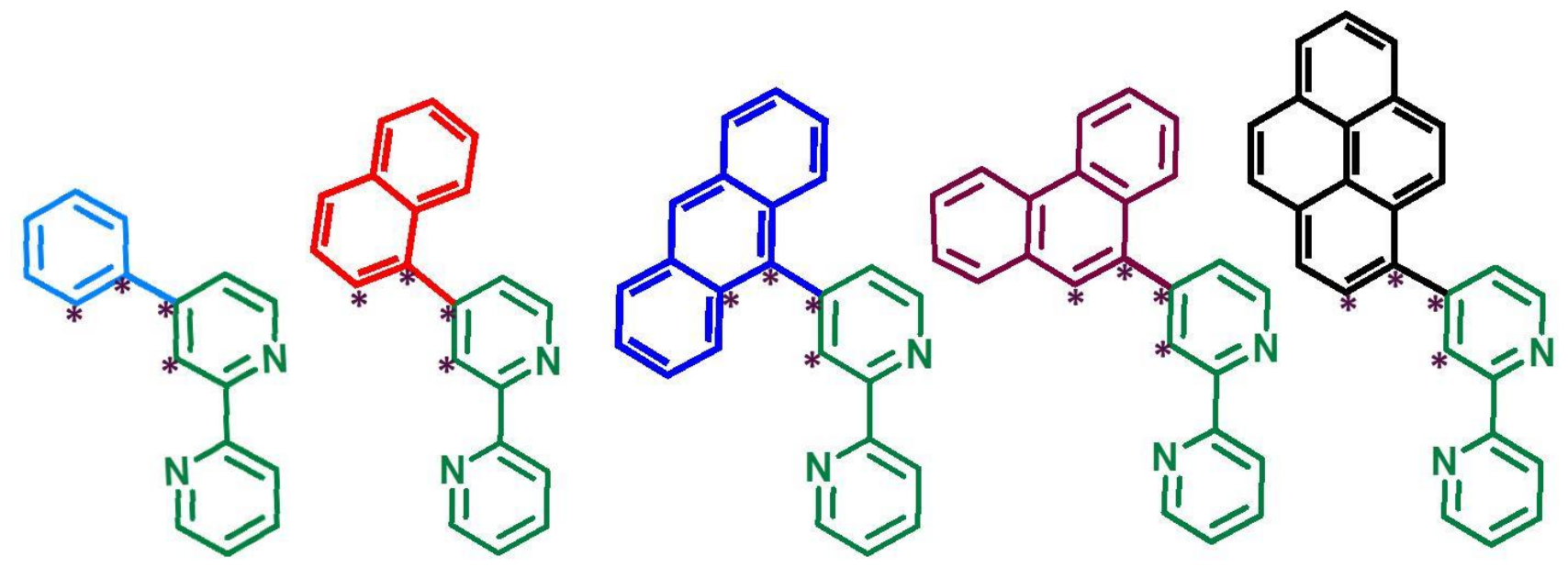

Figure S1. Torsion angle is measured across the atoms labelled in an asterisk (*).

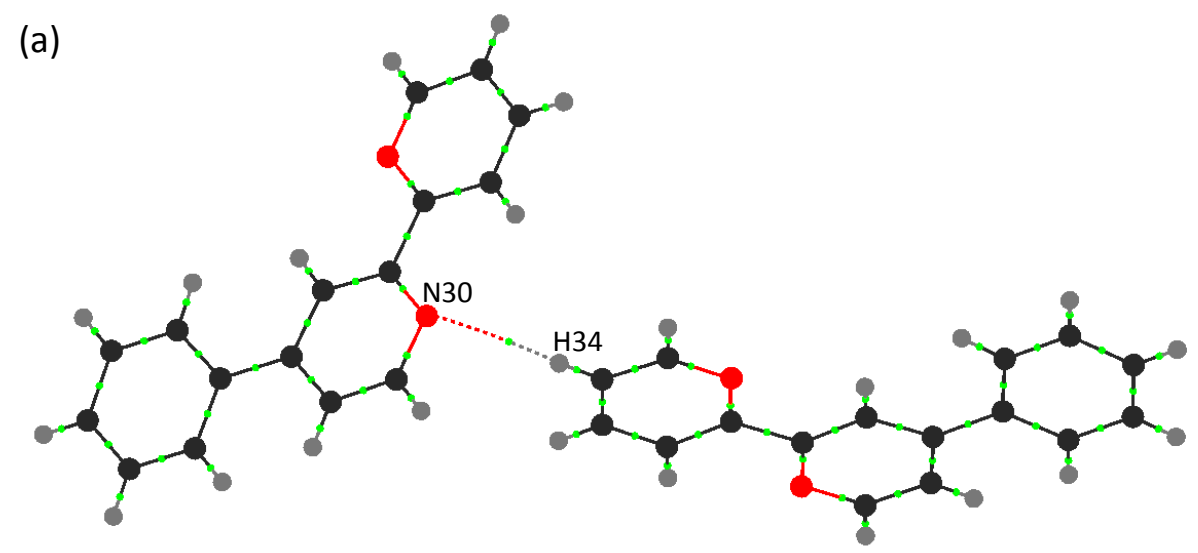




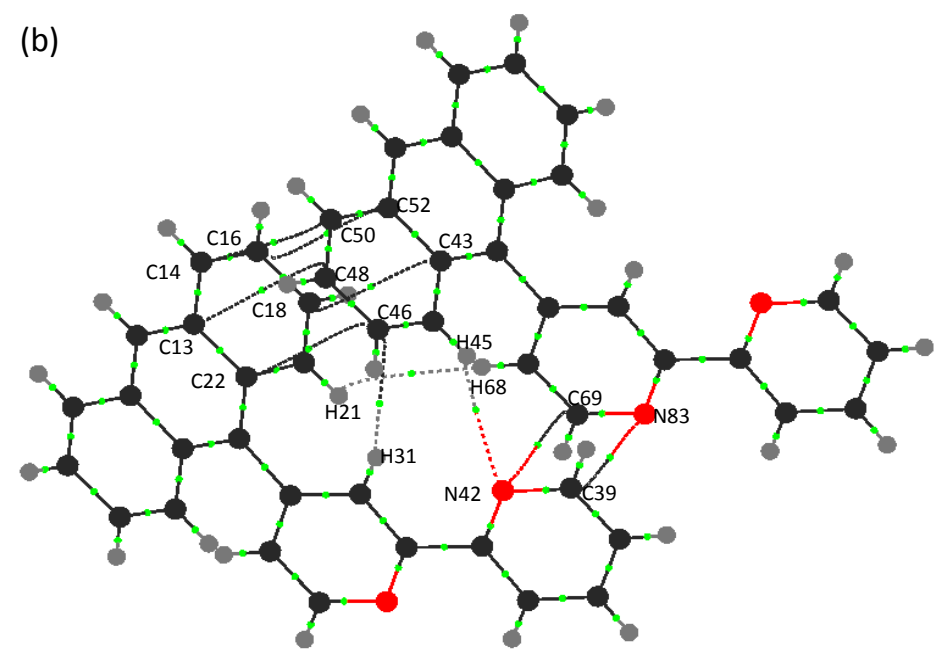

(c)

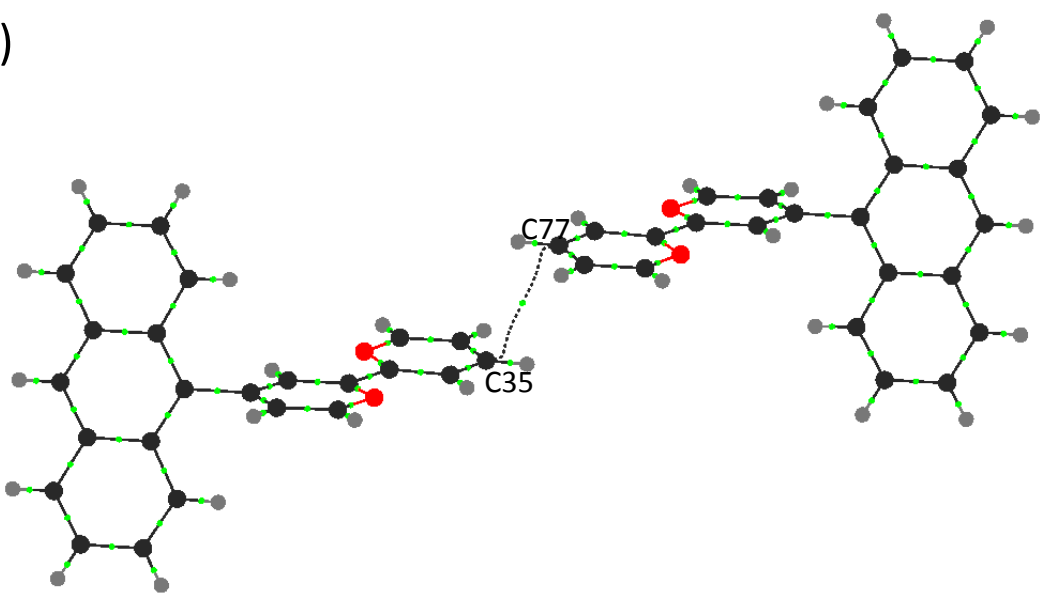

(d)

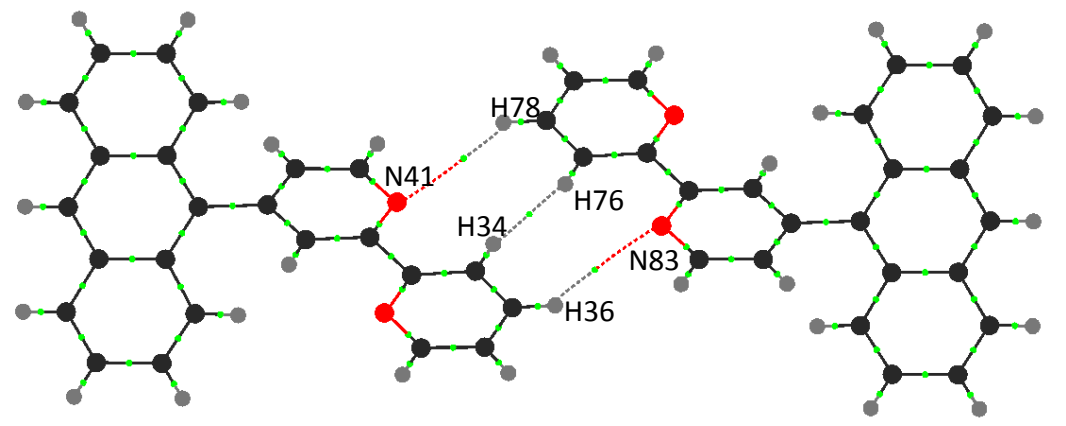


(e)

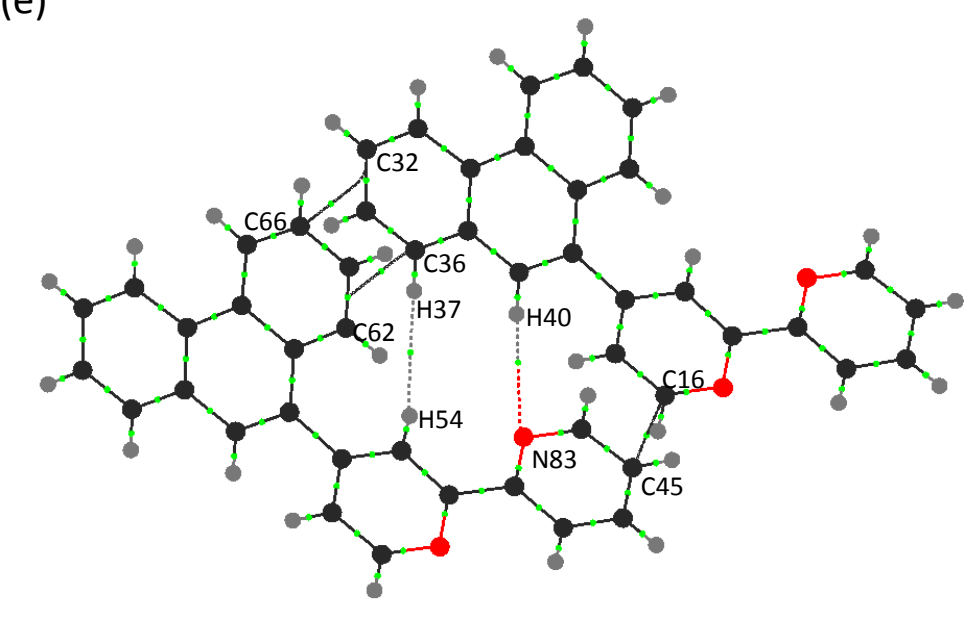

(f)
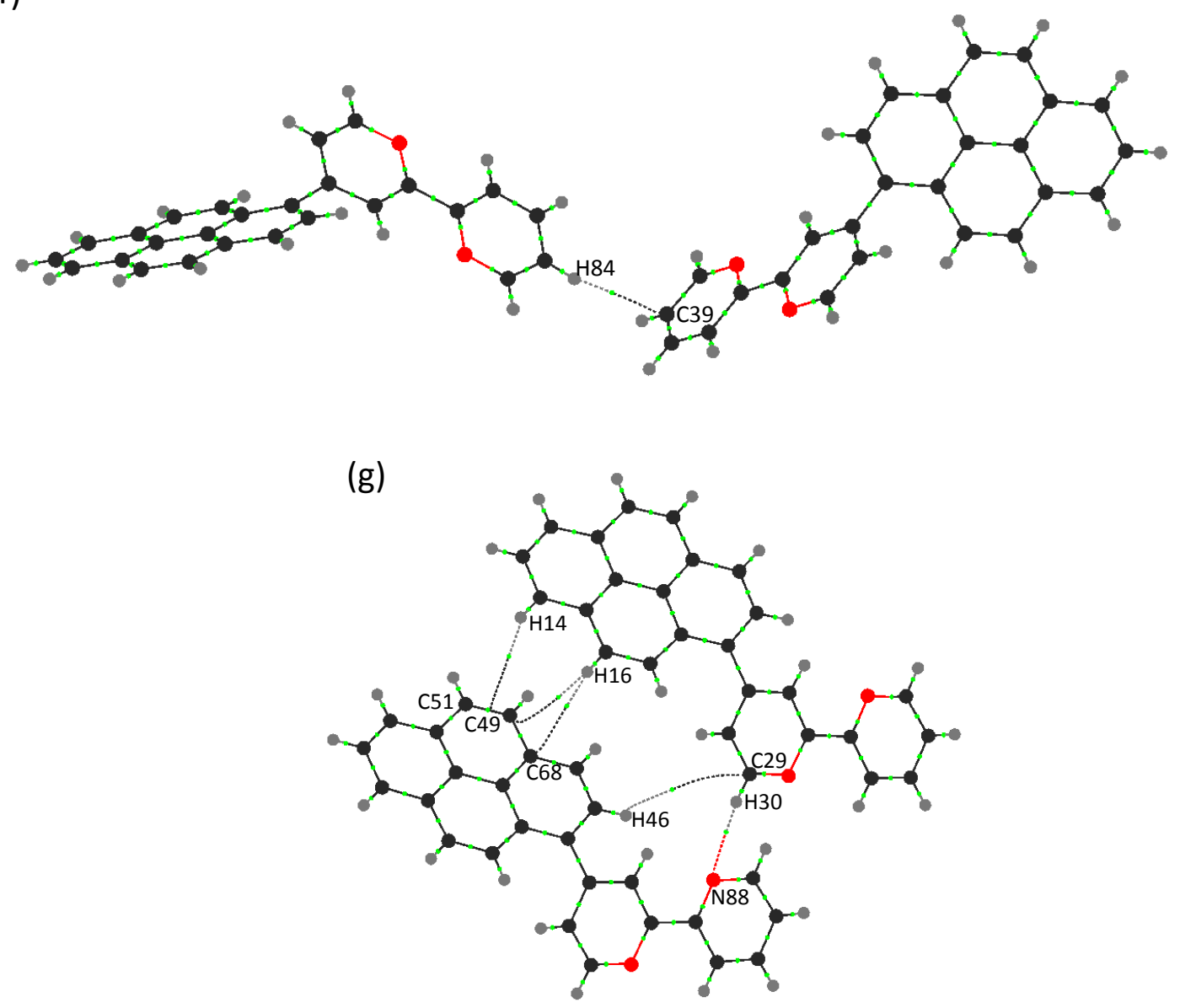

Figure S2. QTAIM electron density maps of (a) $\mathrm{PhB}$, (b) AnBa, (c) AnBb, (d) AnBc, (e) PhenB, (f) $\mathrm{PyBa}$ and (g) $\mathrm{PyBb}$. Dotted lines shown are bond paths and the green dots are bond critical points $(\mathrm{BCP})$. Bond path with a $\mathrm{BCP}$ indicate the existence of intermolecular interactions in the dimers are shown. 

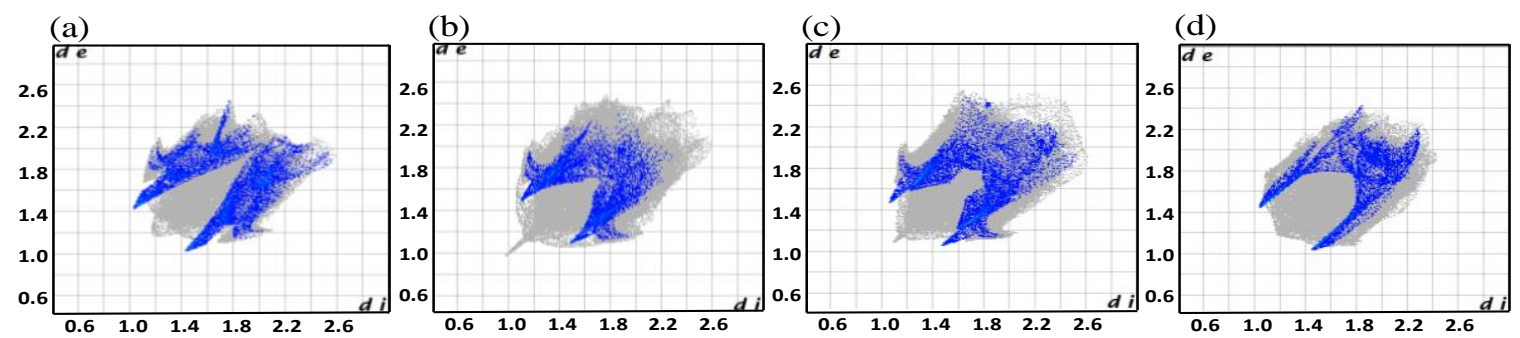

Figure S3. 2D-fingerprint plots representing $\mathrm{C}-\mathrm{H} \bullet \bullet \bullet \mathrm{N}$ interactions in crystalline (a) $\mathrm{PhB}$, (b) AnB, (c) PhenB and (d) PyB obtained from Hirshfeld analyses.
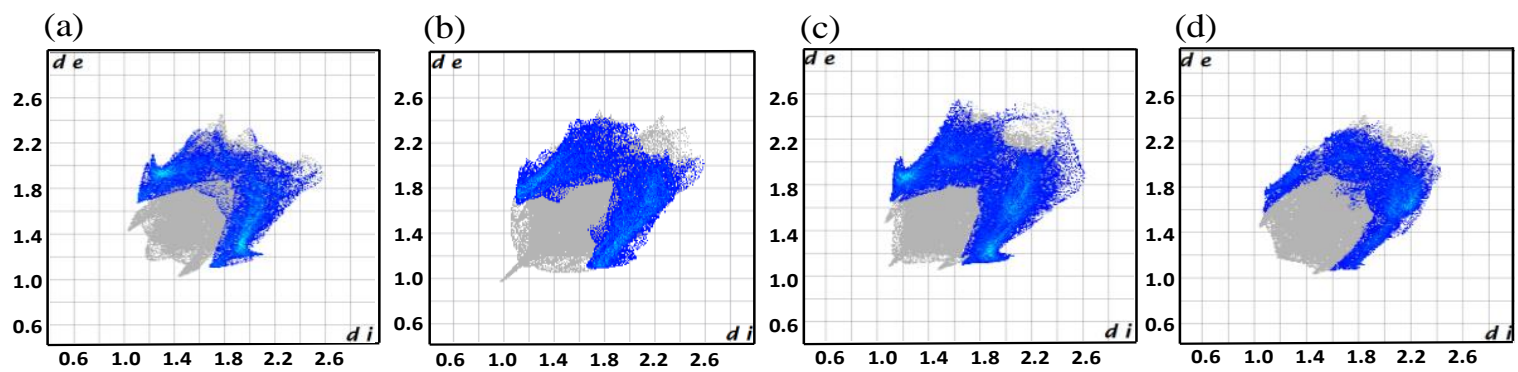

Figure S4. 2D-fingerprint plots representing $\mathrm{C}-\mathrm{H} \bullet \bullet \mathrm{C}$ interactions in crystalline (a) $\mathrm{PhB}$, (b) AnB, (c) PhenB and (d) PyB obtained from Hirshfeld analyses.
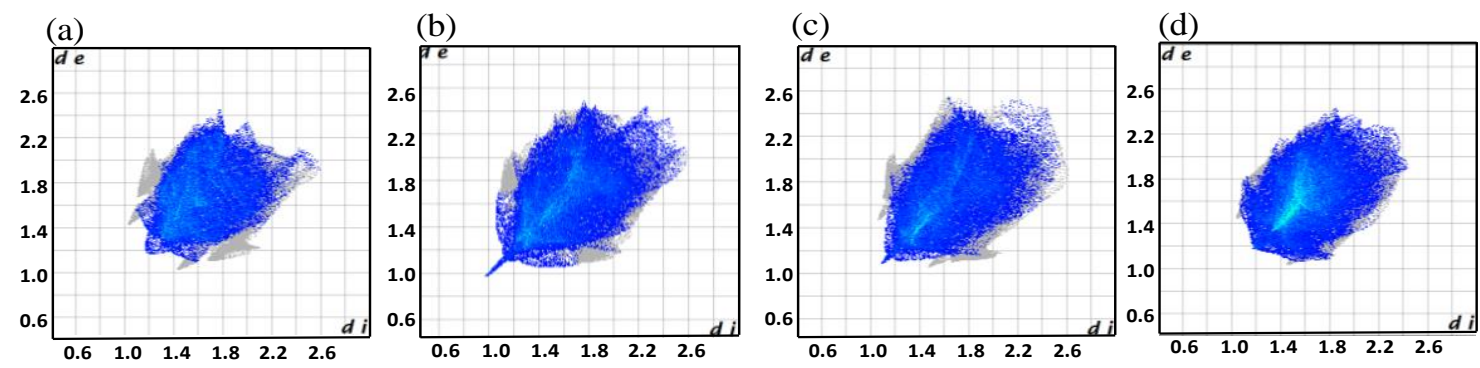

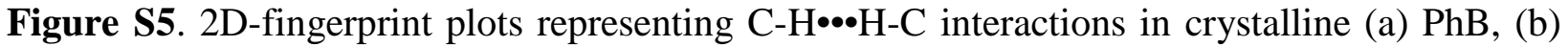
AnB, (c) PhenB and (d) PyB obtained from Hirshfeld analyses.
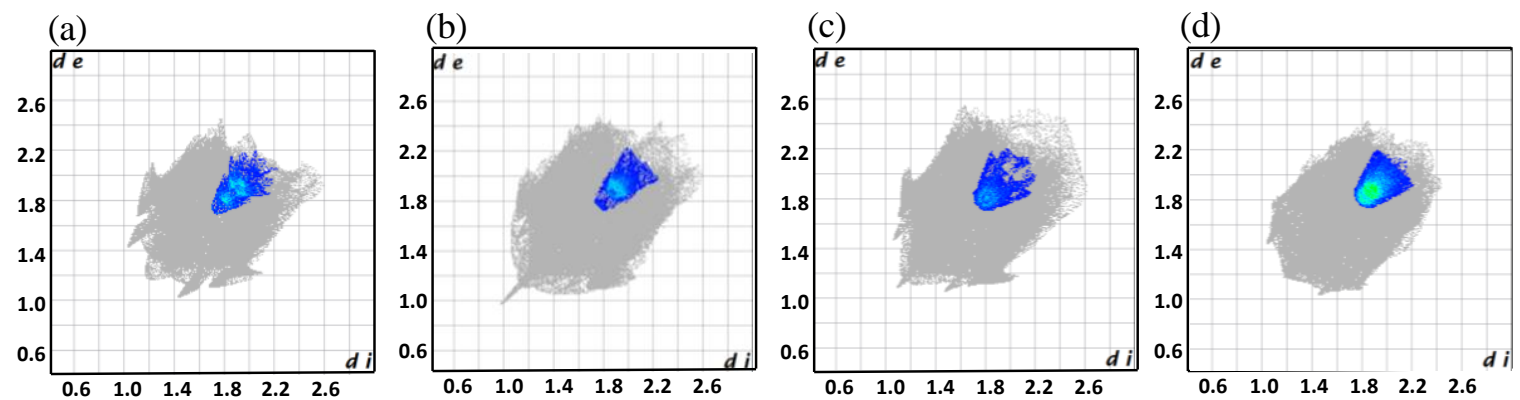

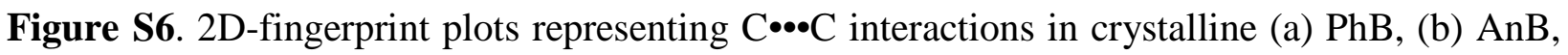
(c) PhenB and (d) PyB obtained from Hirshfeld analyses. 


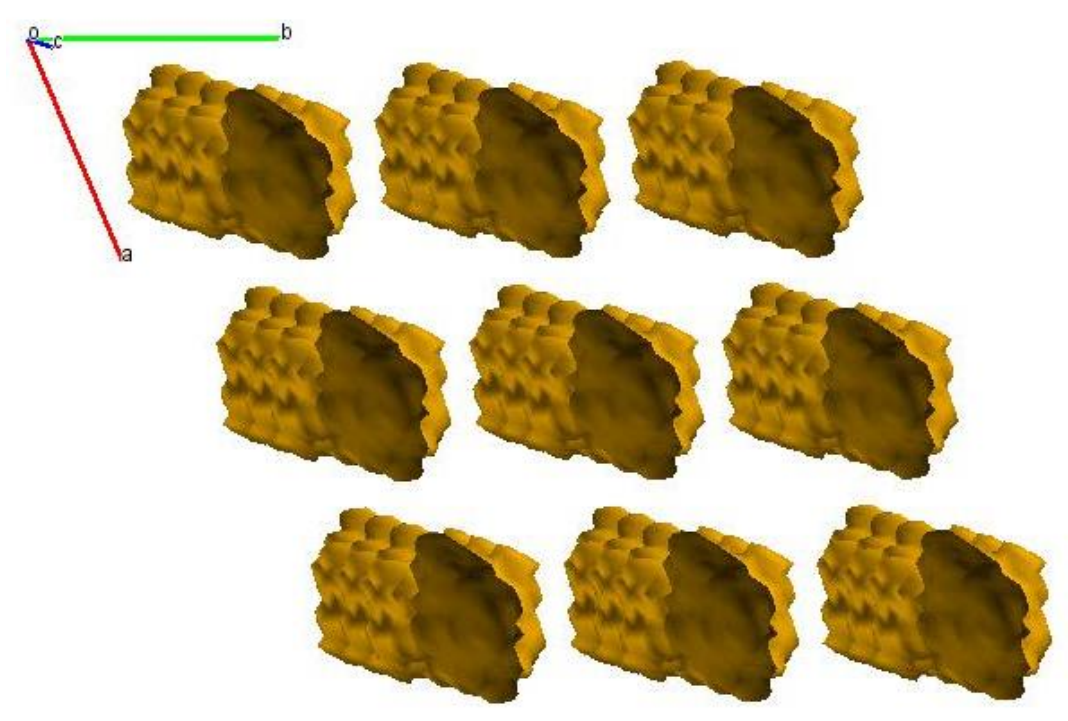

Figure S7. Three dimensional pore distribution in $(\mathrm{NaB})_{2} \bullet \mathrm{D}_{2.5}$ cocrystal.
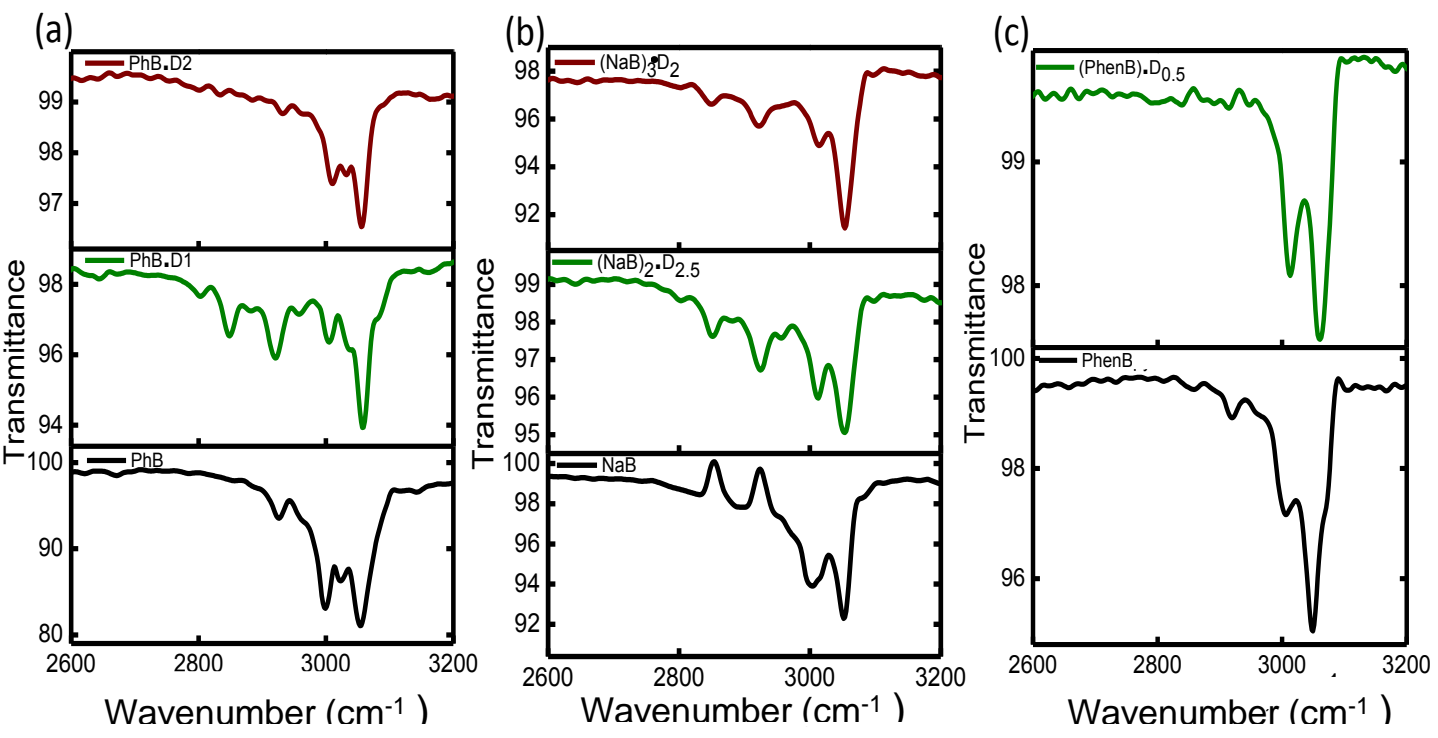

Figure S8. FT-IR spectra of $\mathrm{ArB}$ and $\mathrm{ArB} \cdot \mathrm{D}$. (a) $\mathrm{PhB}, \mathrm{PhB} \cdot \mathrm{D} 1$ and $\mathrm{PhB} \cdot \mathrm{D} 2$, (b) $\mathrm{NaB}$, $(\mathrm{NaB})_{2} \bullet \mathrm{D}_{2.5}$ and $(\mathrm{NaB})_{3} \bullet \mathrm{D}_{2}$ and $(\mathrm{c})$ PhenB and $\mathrm{PhenB} \bullet \mathrm{D}_{0.5}$. 

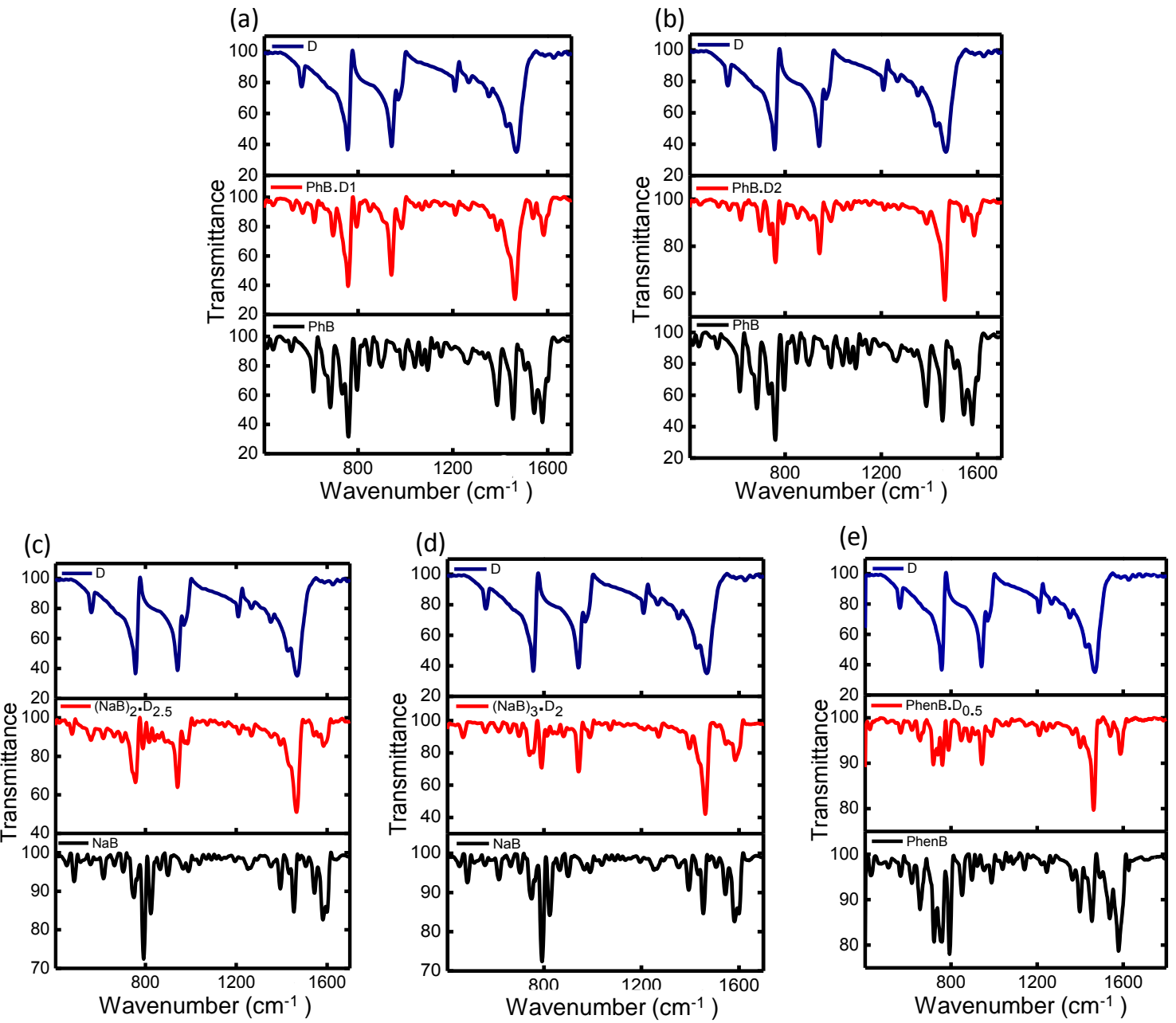

Figure S9. FT-IR spectra of ArB, ArB・D and D. (a) PhB, PhB・D1 and D, (b) $\mathrm{PhB}, \mathrm{PhB} \bullet \mathrm{D} 2$ and D, (c) NaB, $(\mathrm{NaB})_{2} \bullet \mathrm{D}_{2.5}$ and $\mathrm{D},(\mathrm{d}) \mathrm{NaB},(\mathrm{NaB})_{3} \bullet \mathrm{D}_{2}$ and $\mathrm{D}$ and (e) PhenB, PhenB $\bullet \mathrm{D}_{0.5}$ and $\mathrm{D}$. 
(a)

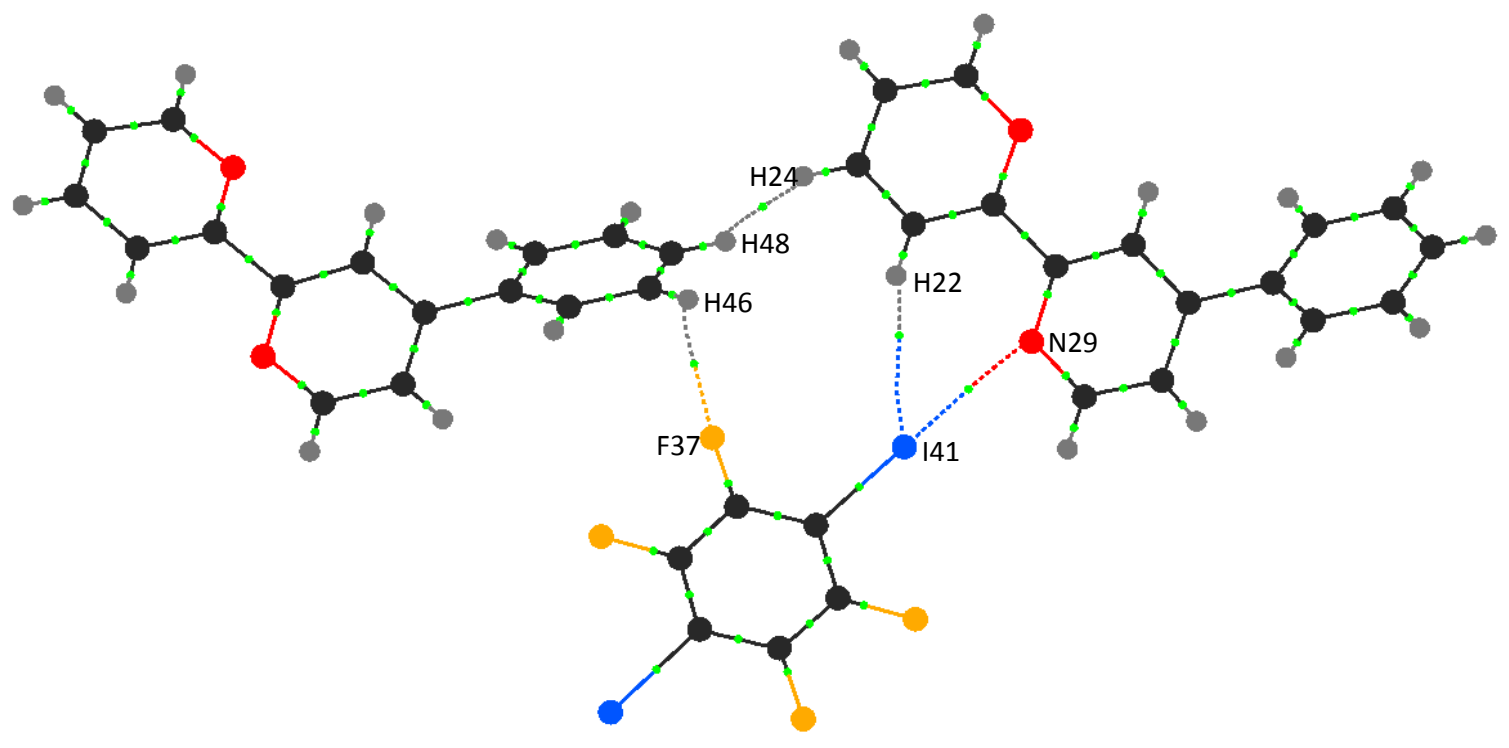

(b)

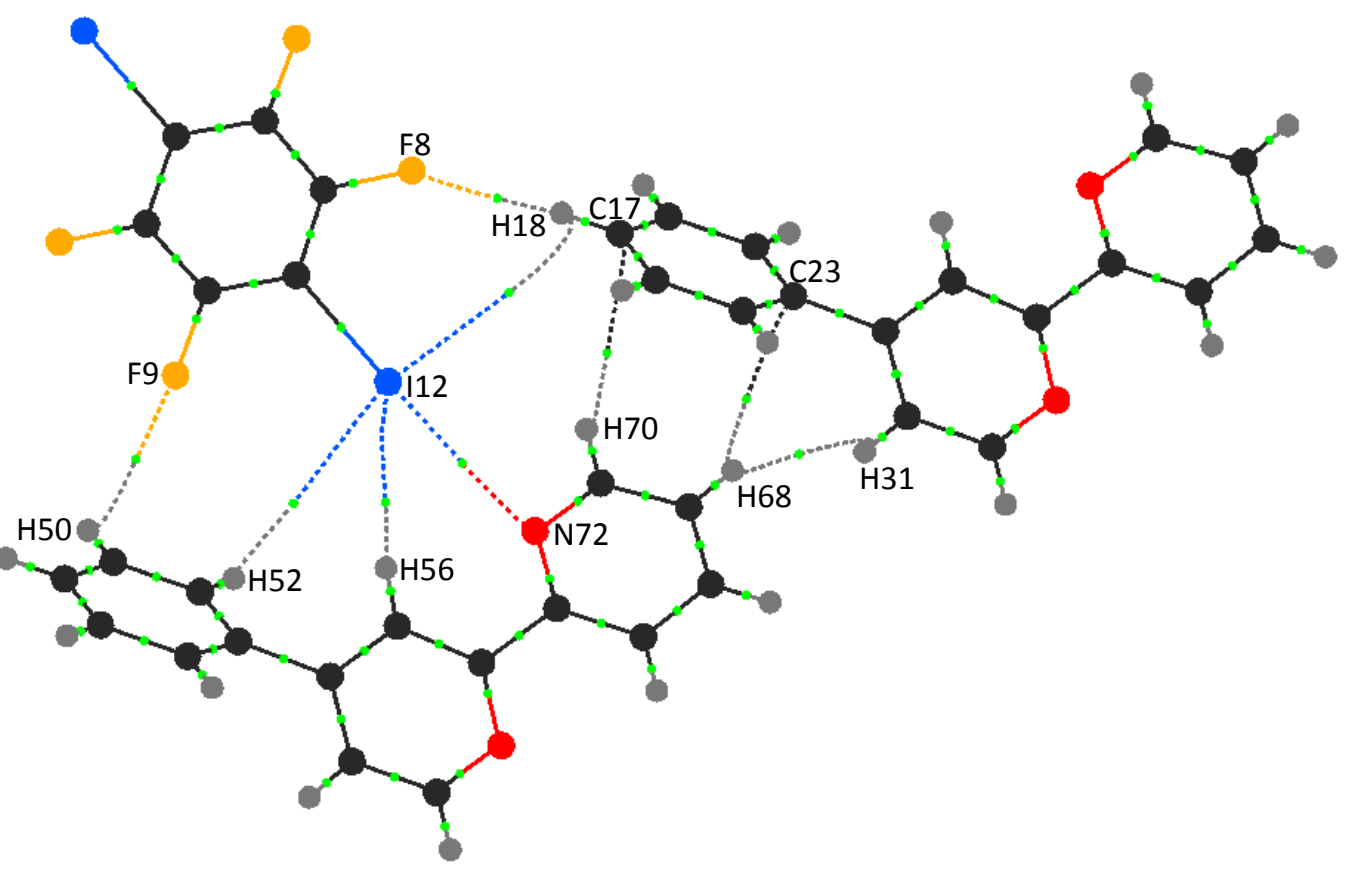


(c)
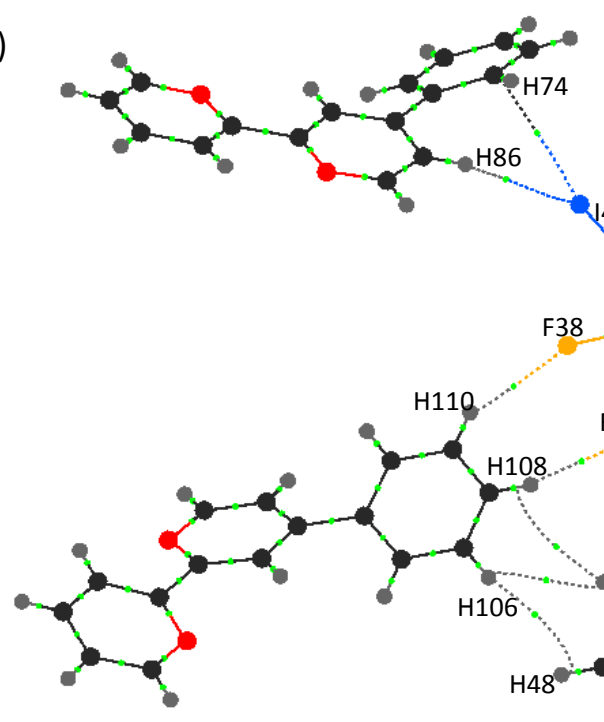

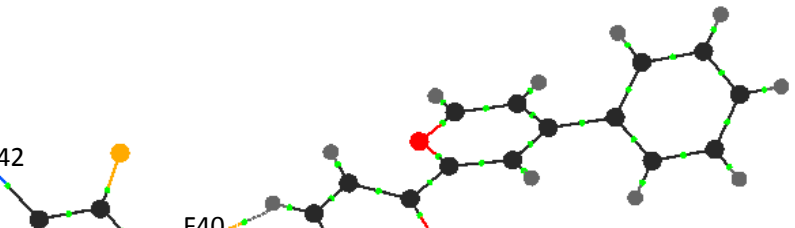

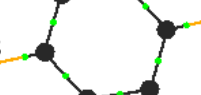

F40
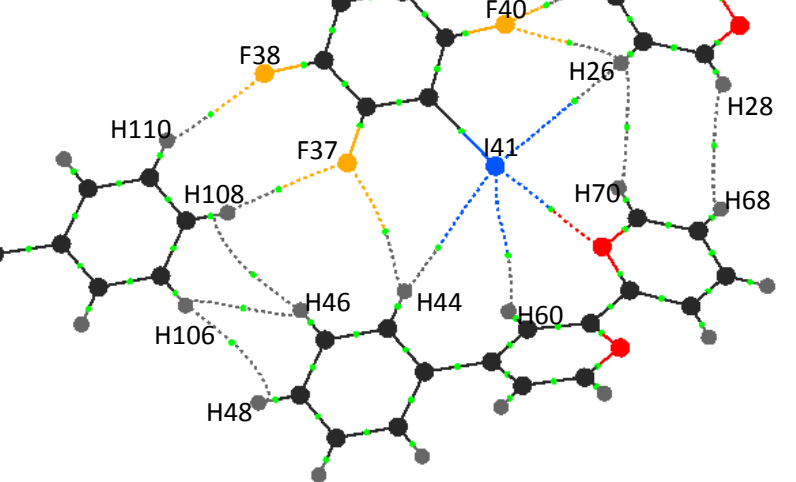

106 
(d)

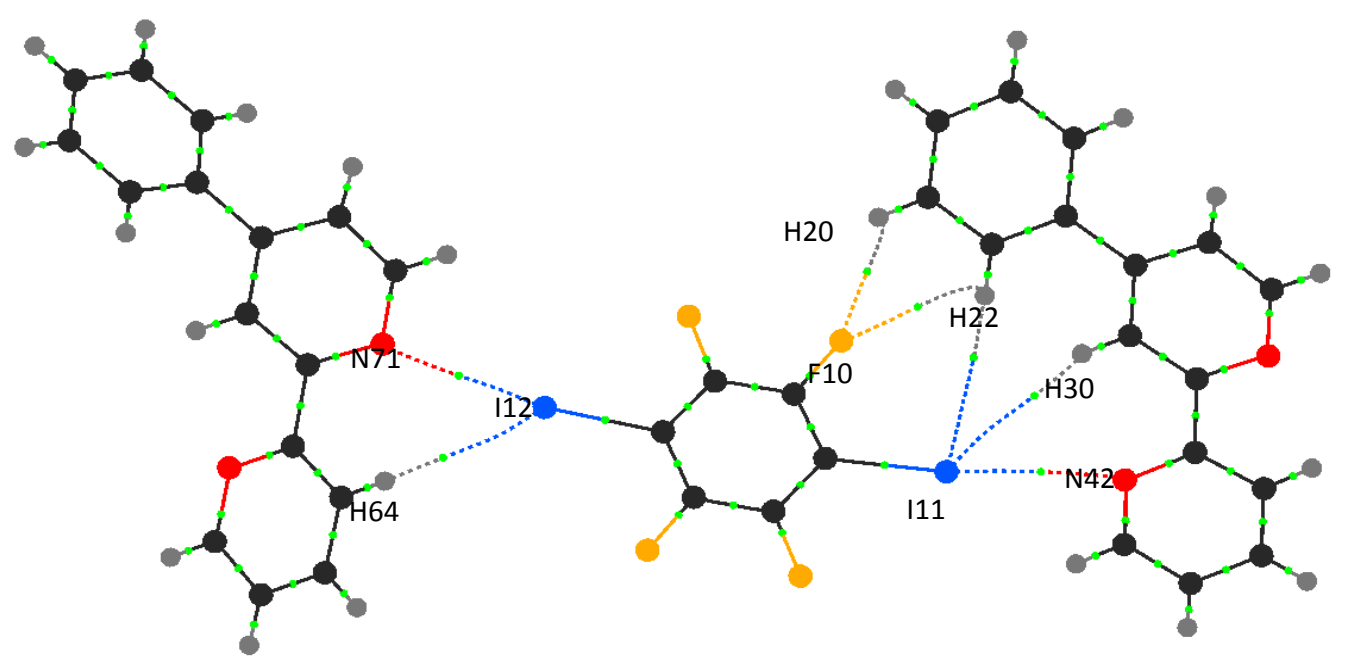

(e)

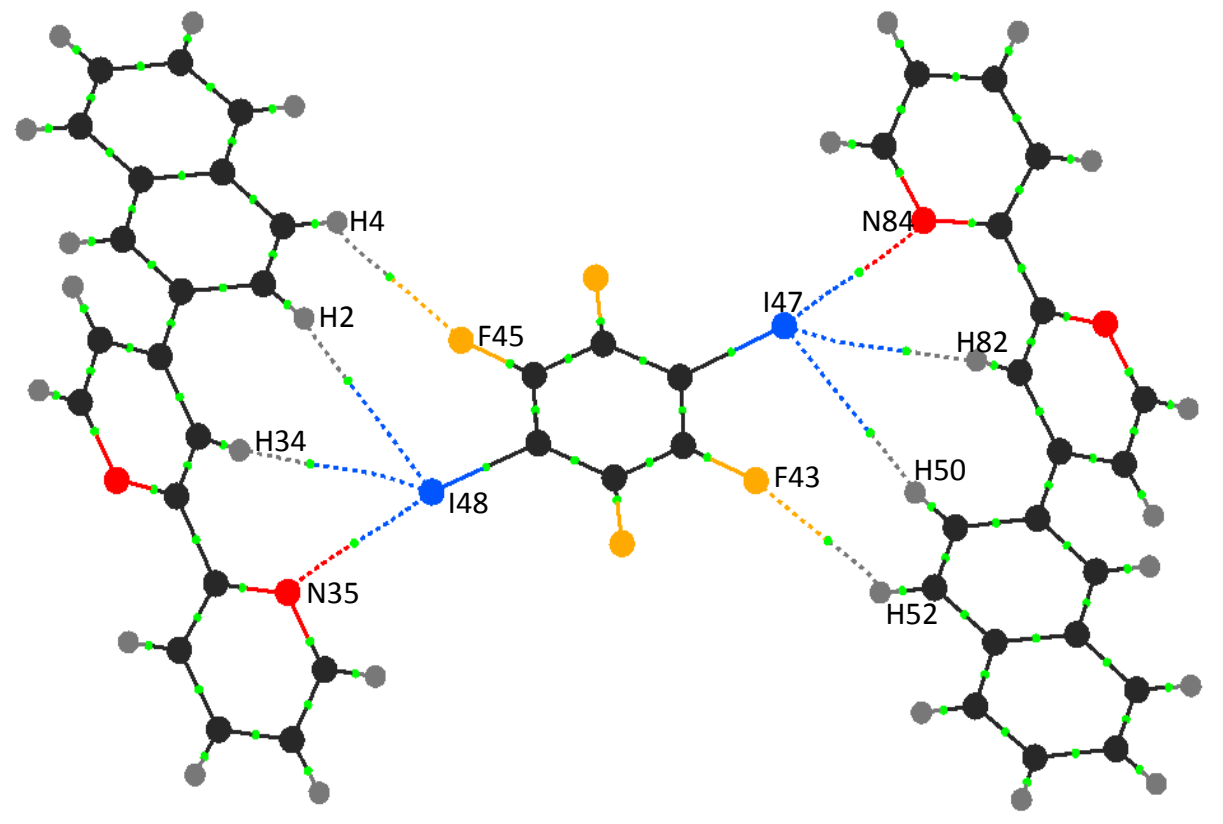




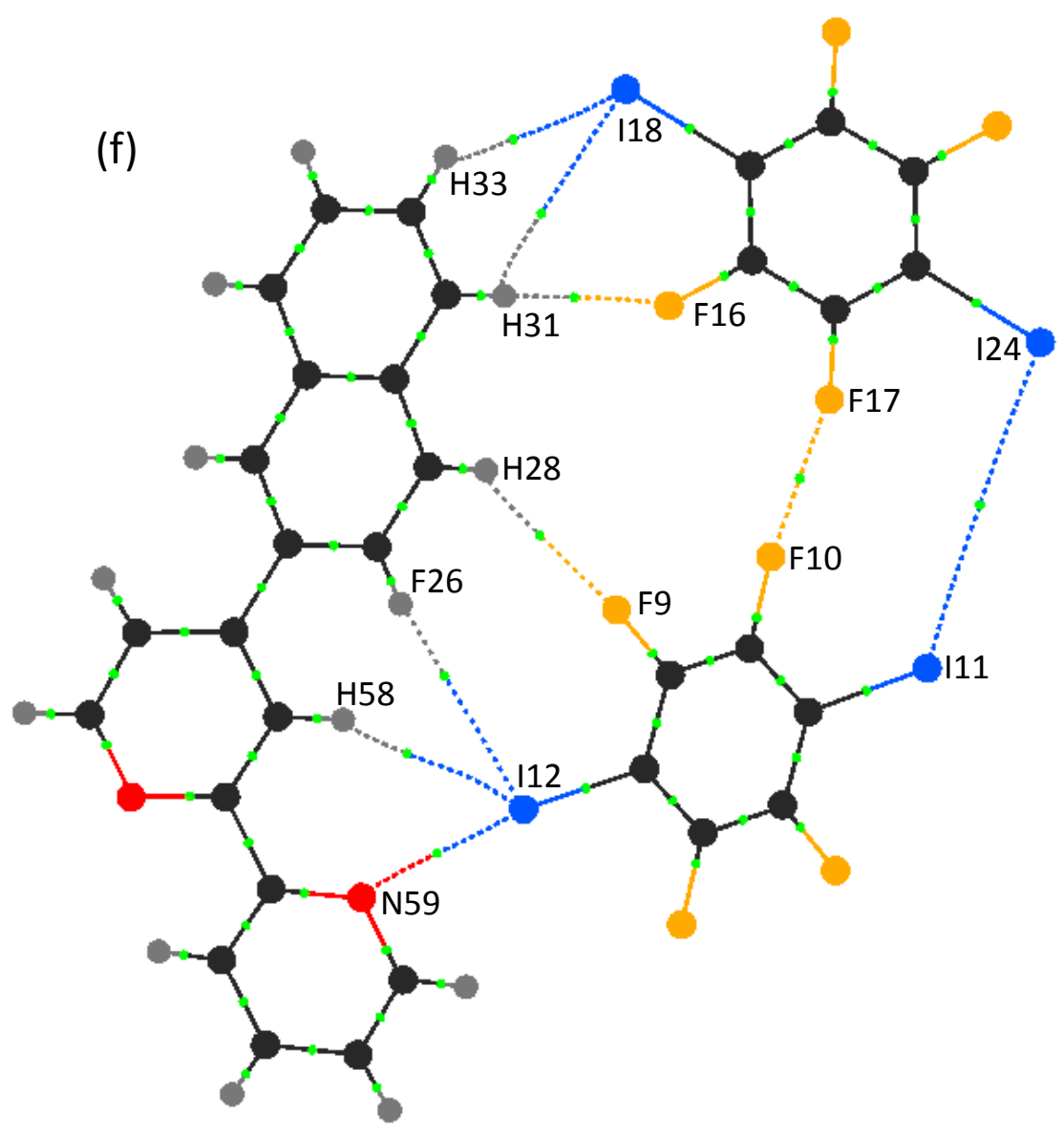

(g)

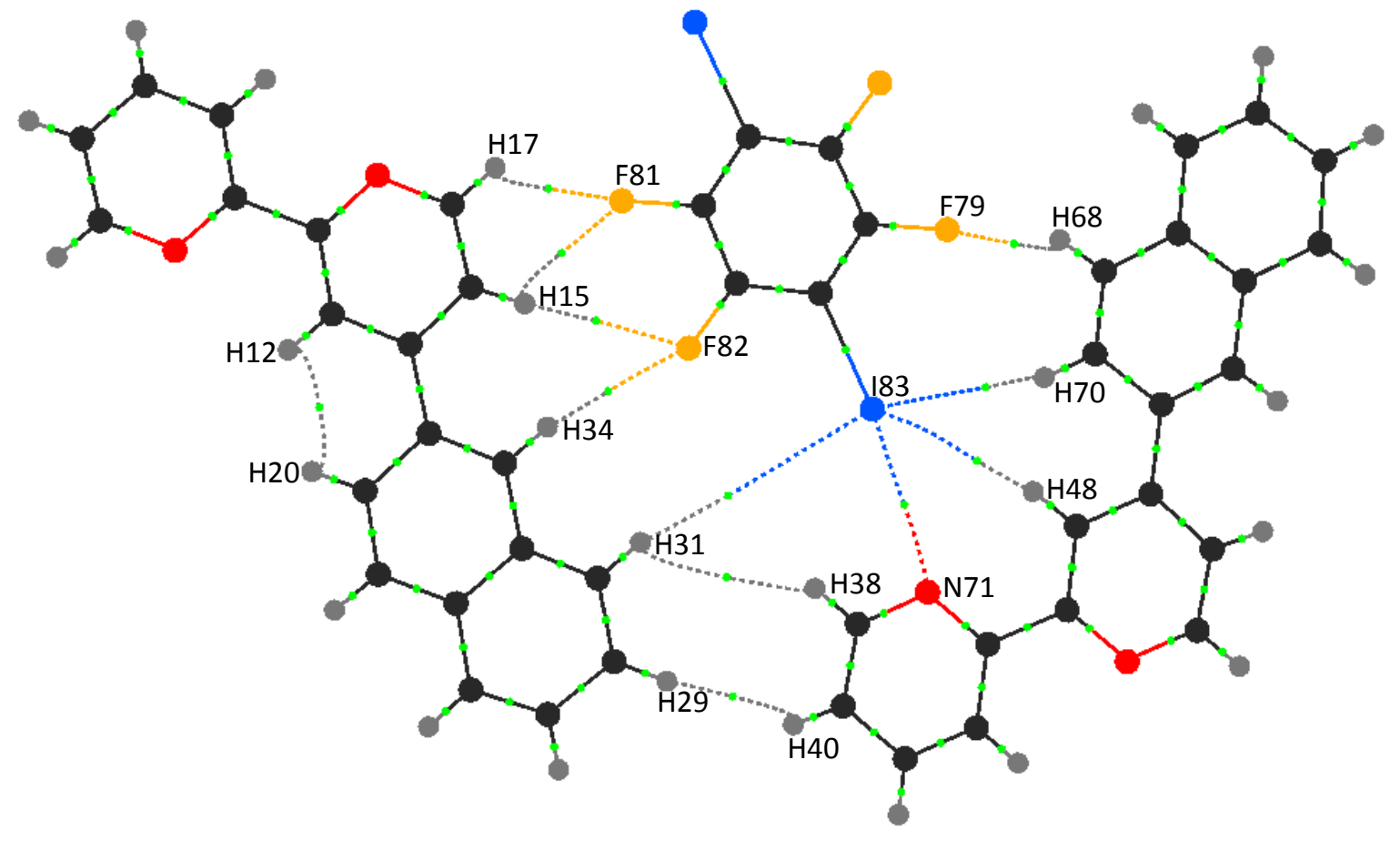


(h)

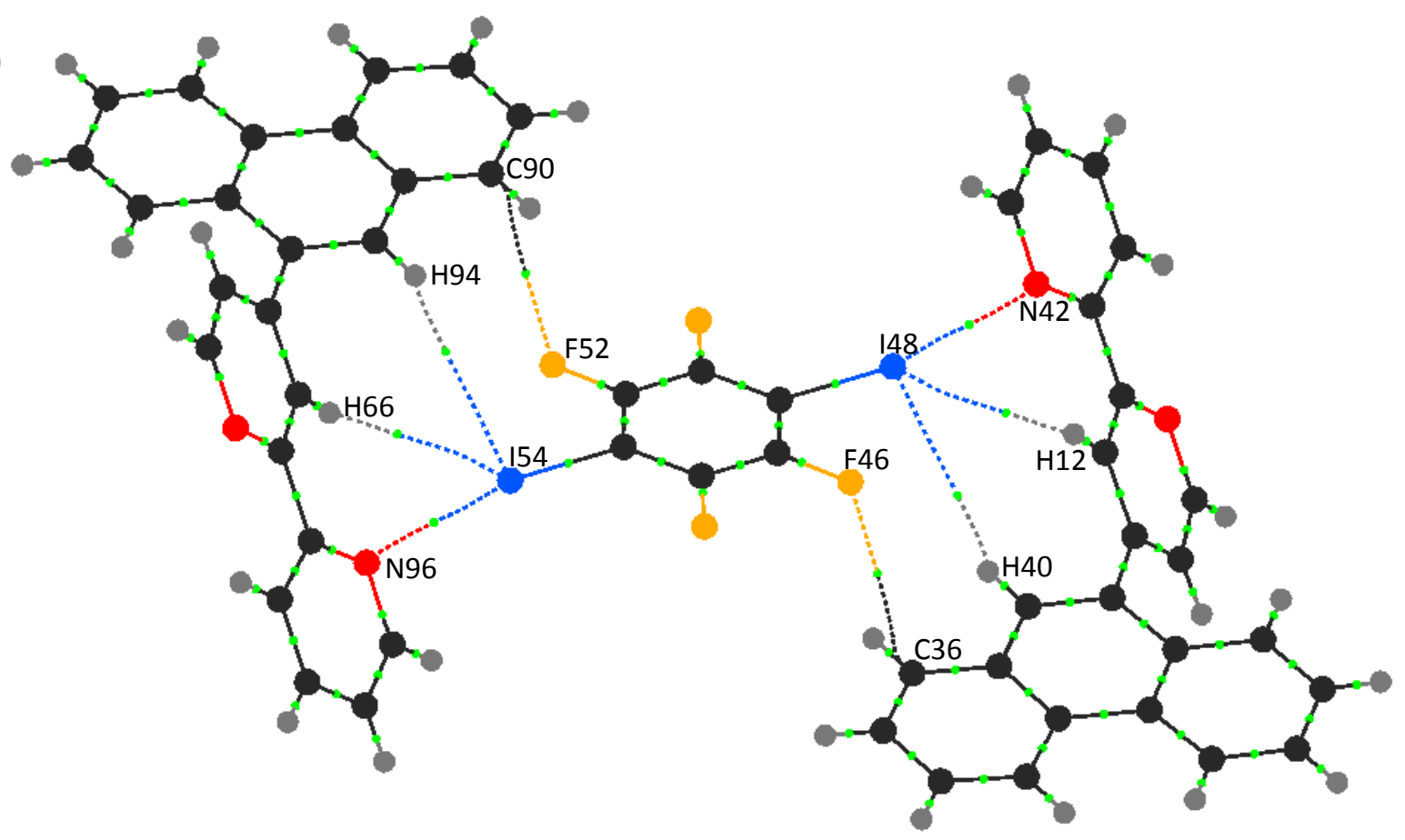

(i)

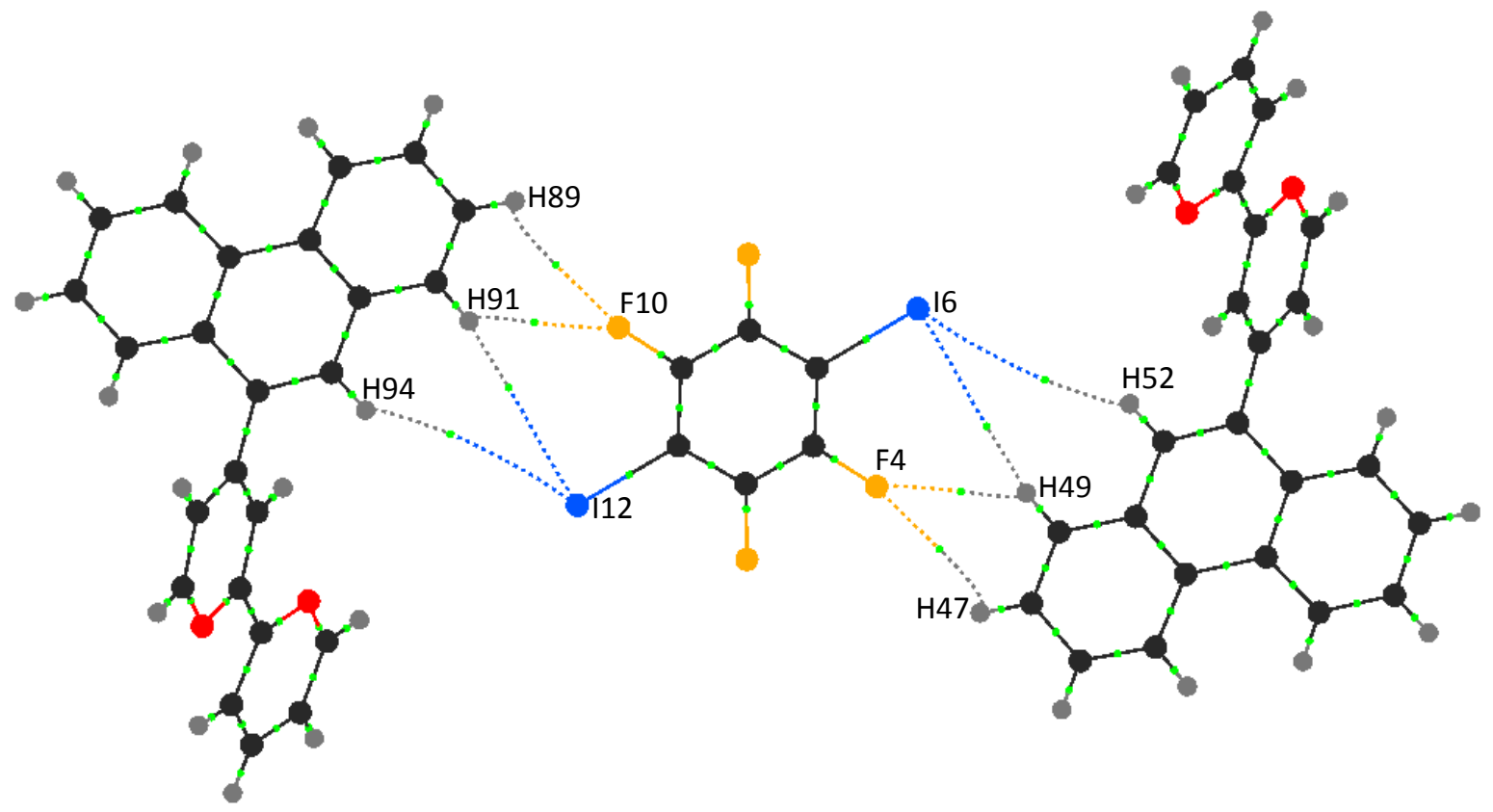

Figure S10. QTAIM electron density maps of (a) $\mathrm{PhB} \cdot \mathrm{D} 1 \mathrm{a}$, (b) $\mathrm{PhB} \cdot \mathrm{D} 1 \mathrm{~b}$, (c) $\mathrm{PhB} \cdot \mathrm{D} 2 \mathrm{a}$, (d) $\mathrm{PhB} \cdot \mathrm{D} 2 \mathrm{~b}$ ，(e) $(\mathrm{NaB})_{2} \cdot \mathrm{D}_{2.5} \mathrm{a}$ ，(f) $(\mathrm{NaB})_{2} \cdot \mathrm{D}_{2.5} \mathrm{~b}$ ，(g) (NaB) $)_{3} \cdot \mathrm{D}_{2} \mathrm{a}$, (h) PhenB $\mathrm{D}_{0.5} \mathrm{a}$ and (i) PhenB $\cdot \mathrm{D}_{0.5} \mathrm{~b}$. Dotted lines shown are bond paths and the green dots are bond critical points (BCP). Bond path with a $\mathrm{BCP}$ indicate the existence of intermolecular interactions in different molecules. 
(a)

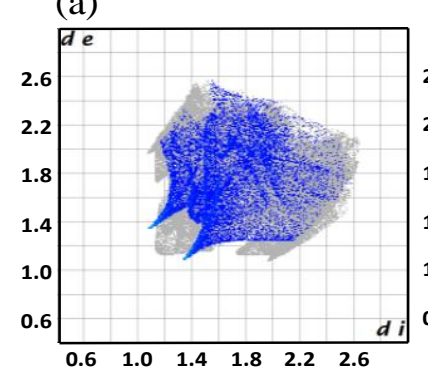

(b)

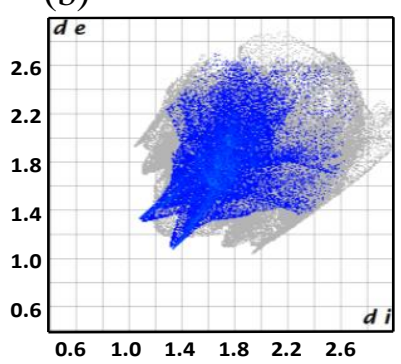

(e)

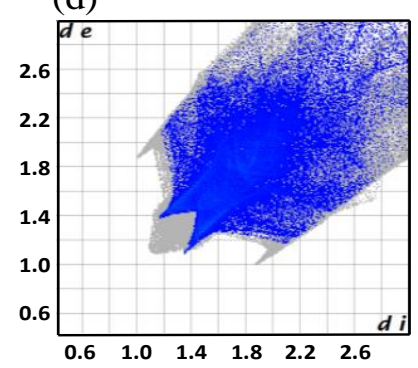

(c)
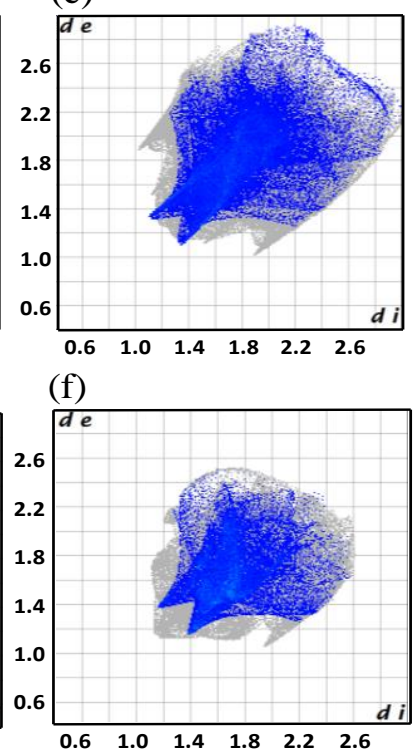

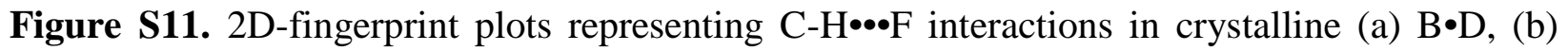
$\mathrm{PhB} \bullet \mathrm{D} 1$, (c) $\mathrm{PhB} \bullet \mathrm{D} 2$, (d) $(\mathrm{NaB})_{2} \bullet \mathrm{D}_{2.5}$, (e) $(\mathrm{NaB})_{3} \bullet \mathrm{D}_{2}$ and (f) PhenB $\bullet \mathrm{D}_{0.5}$ obtained from Hirshfeld analyses.

(a)

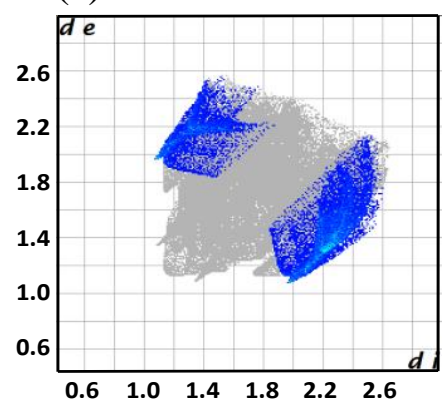

(d)

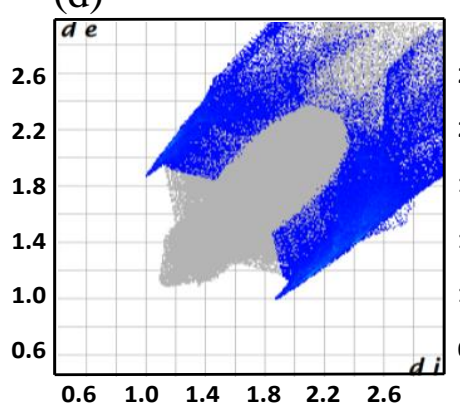

(b)

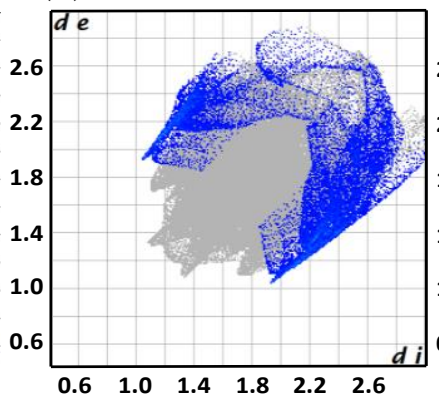

(e)

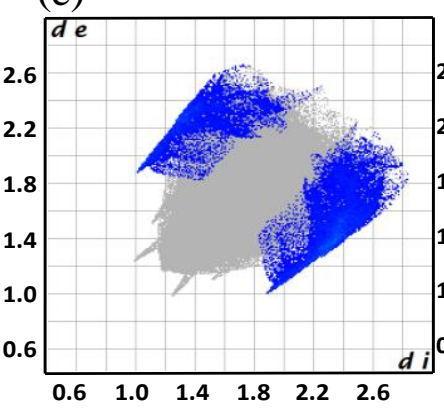

(c)

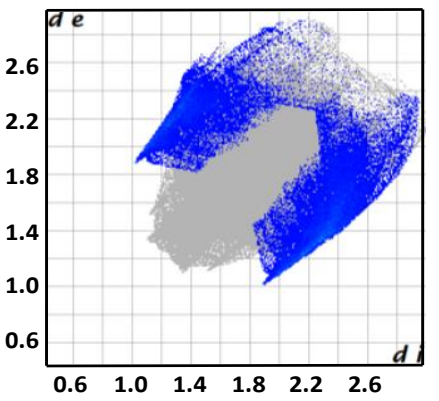

(f)

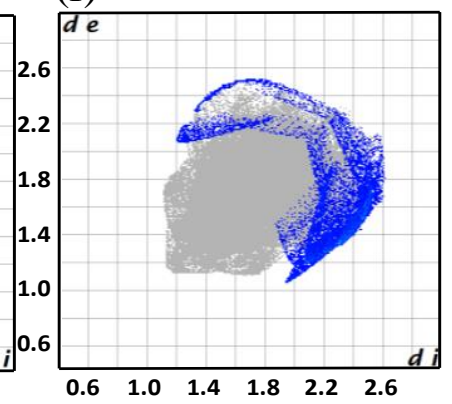

Figure S12. 2D-fingerprint plots representing $\mathrm{C}-\mathrm{H} \bullet \bullet \bullet I$ interactions in crystalline (a) B•D, (b) $\mathrm{PhB} \bullet \mathrm{D} 1$, (c) $\mathrm{PhB} \bullet \mathrm{D} 2$, (d) $(\mathrm{NaB})_{2} \bullet \mathrm{D}_{2.5}$, (e) $(\mathrm{NaB})_{3} \bullet \mathrm{D}_{2}$ and (f) PhenB $\bullet \mathrm{D}_{0.5}$ obtained from Hirshfeld analyses. 

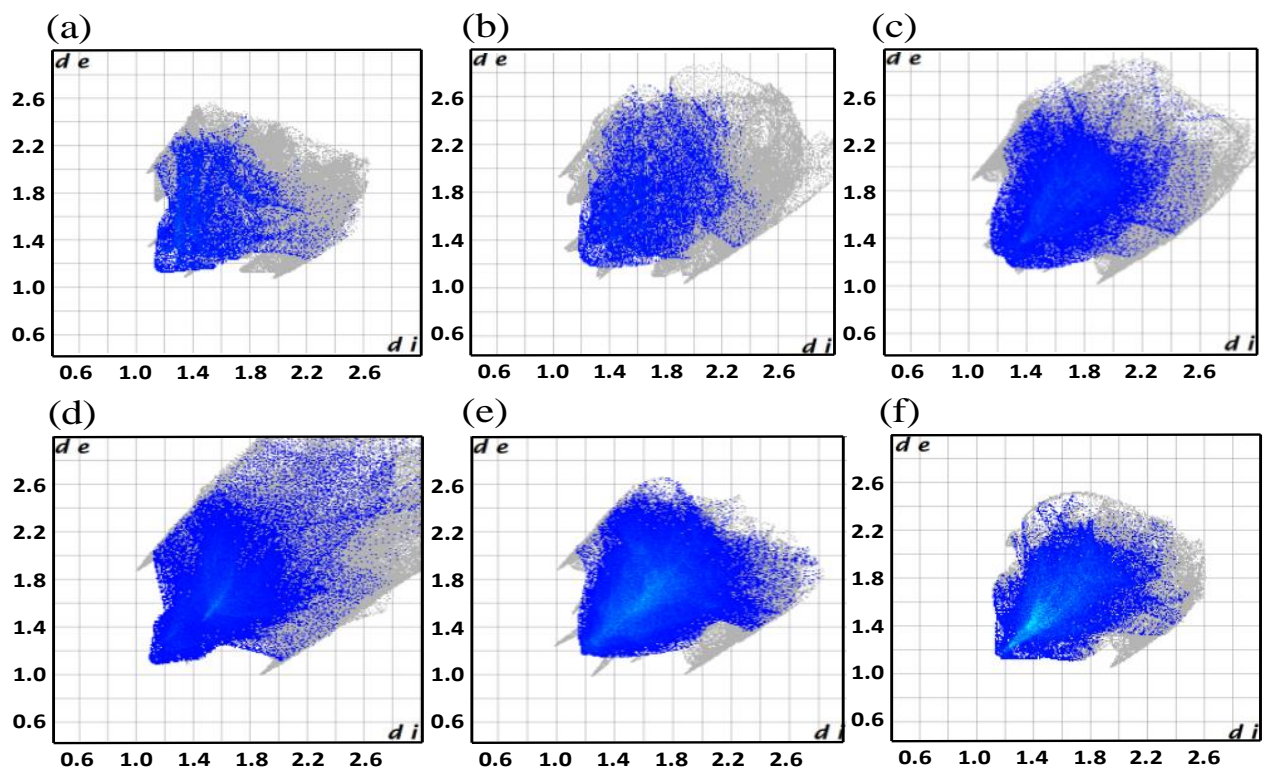

Figure S13. 2D-fingerprint plots representing $\mathrm{C}-\mathrm{H} \bullet \bullet \cdot \mathrm{H}-\mathrm{C}$ interactions in crystalline (a) $\mathrm{B} \bullet \mathrm{D}$, (b) $\mathrm{PhB} \bullet \mathrm{D} 1$, (c) $\mathrm{PhB} \bullet \mathrm{D} 2$, (d) $(\mathrm{NaB})_{2} \bullet \mathrm{D}_{2.5}$, (e) $(\mathrm{NaB})_{3} \bullet \mathrm{D}_{2}$ and (f) PhenB $\bullet \mathrm{D}_{0.5}$ obtained from Hirshfeld analyses.

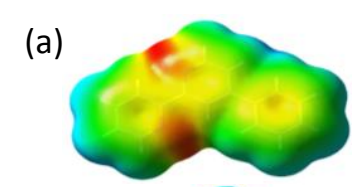

(b)

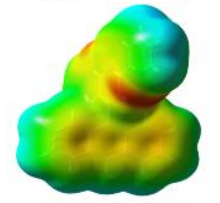

(c)

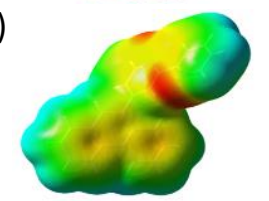

(d)

(e)

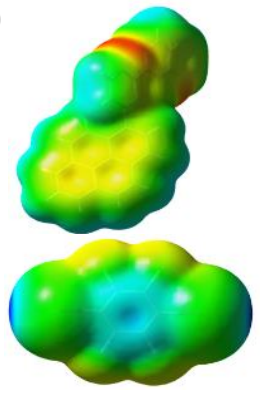

(f)

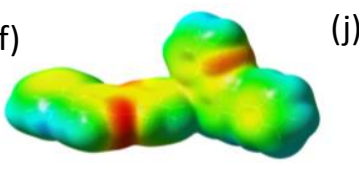

(g)

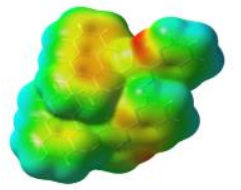

(h)

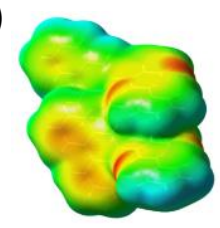

(i)

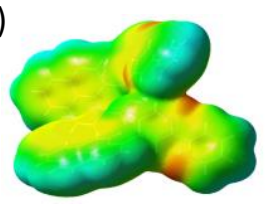

(j)

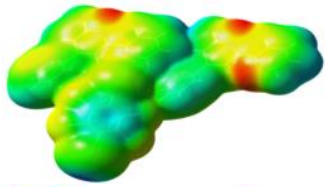

(k)

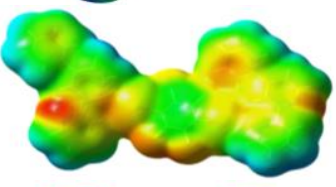

(I)

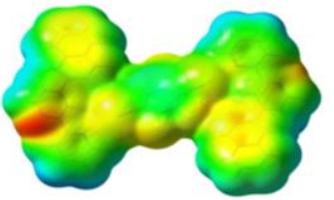

(m)

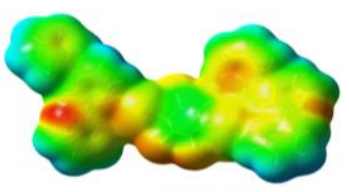

(n)

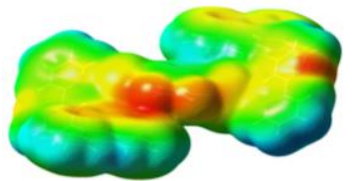

Figure S14. Electrostatic surface potential (ESP) map of (a-d) ArB monomers, (f-i) dimers and (j-n) cocrystals. ESP maps of (a) PhB, (b) AnB, (c) PhenB and (d) PyB monomers. ESP map of (e) $\mathrm{D}$, (f) $\mathrm{PhB}$, (g) AnB, (h) PhenB and (i) PyB dimers. ESP map of (j) PhB $\bullet$ D1, (k) PhB $\bullet$ 2 2, (1) $(\mathrm{NaB})_{2} \cdot \mathrm{D}_{2.5},(\mathrm{~m})(\mathrm{NaB})_{3} \cdot \mathrm{D}_{2}$ and $(\mathrm{n}) \mathrm{PhenB} \cdot \mathrm{D}_{0.5}$ cocrystals. 


\section{References:}

1. Bader, R. F. W.; Atoms in Molecules: A Quantum Theory. Oxford University Press: Oxford, U.K., 1990.

2. Frisch, M. J.; Trucks, G. W.; Schlegel, H. B.; Scuseria, G. E.; Robb, M. A.; Cheeseman, J. R.; Scalmani, G.; Barone, V.; Mennucci, B.; Petersson, G. A.; Nakatsuji, H.; Caricato, M.; Li, X.; Hratchian, H. P.; Izmaylov, A. F.; Bloino, J.; Zheng, G.; Sonnenberg, J. L.; Hada, M.; Ehara, M.; Toyota, K.; Fukuda, R.; Hasegawa, J.; Ishida, M.; Nakajima, T.; Honda, Y.; Kitao, O.; Nakai, H.; Vreven, T.; Montgomery Jr., J. A.; Peralta, J. E.; Ogliaro, F.; Bearpark, M. J.; Heyd, J.; Brothers, E. N.; Kudin, K. N.; Staroverov, V. N.; Kobayashi, R.; Normand, J.; Raghavachari, K.; Rendell, A. P.; Burant, J. C.; Iyengar, S. S.; Tomasi, J.; Cossi, M.; Rega, N.; Millam, N. J.; Klene, M.; Knox, J. E.; Cross, J. B.; Bakken, V.; Adamo, C.; Jaramillo, J.; Gomperts, R.; Stratmann, R. E.; Yazyev, O.; Austin, A. J.; Cammi, R.; Pomelli, C.; Ochterski, J. W.; Martin, R. L.; Morokuma, K.; Zakrzewski, V. G.; Voth, G. A.; Salvador, P.; Dannenberg, J. J.; Dapprich, S.; Daniels, A. D.; Farkas, Ö.; Foresman, J. B.; Ortiz, J. V.; Cioslowski, J.; Fox, D. J. Gaussian 09; Gaussian, Inc.: Wallingford, CT, USA, 2009.

3. Yang, Y.; Weaver, M. N.; Merz, K. M., J. Phys. Chem. A 2009, 113, 9843.

4. Yahia-Ouahmed, M.; Tognetti, V.; Joubert, L., J. Chem. Theory Comput. 2015, 1053, 254.

5. Blanco, M. A.; Martín Pendás, A.; Francisco, E., J. Chem. Theory Comput. 2005, 1, 1096.

6. Martín Pendás, A.; Blanco, M. A.; Francisco, E., J. Chem. Phys. 2006, 125, 184112.

7. Wang, H.; Wang, W.; Jin, W. J., Chem. Rev. 2016, 116, 5072.

8. Wolff, S. K.; Grinwood, D. J.; McKinnon, J. J.; Turner, M. J.; Jayatilaka, D.; Spackman, M. A. CrystalExplorer 3.0; University of Western Australia, Perth, Australia, 2012.

9. Kodama, K.; Kobayashi, A.; Hirose, T., Tetrahedron Lett. 2013, 54, 5514.

10. Gavezzotti, A., Acc. Chem. Res. 1994, 27, 309. 